\title{
Information-based detection of nonlinear Granger causality in multivariate processes via a nonuniform embedding technique
}

\author{
Luca Faes, ${ }^{1, *}$ Giandomenico Nollo, ${ }^{1}$ and Alberto Porta ${ }^{2}$ \\ ${ }^{1}$ Department of Physics and BIOtech, University of Trento, Trento, Italy \\ ${ }^{2}$ Galeazzi Orthopaedic Institute, Department of Technologies for Health, University of Milan, Milan, Italy \\ (Received 17 September 2010; revised manuscript received 7 February 2011; published 11 May 2011)
}

\begin{abstract}
We present an approach, framed in information theory, to assess nonlinear causality between the subsystems of a whole stochastic or deterministic dynamical system. The approach follows a sequential procedure for nonuniform embedding of multivariate time series, whereby embedding vectors are built progressively on the basis of a minimization criterion applied to the entropy of the present state of the system conditioned to its past states. A corrected conditional entropy estimator compensating for the biasing effect of single points in the quantized hyperspace is used to guarantee the existence of a minimum entropy rate at which to terminate the procedure. The causal coupling is detected according to the Granger notion of predictability improvement, and is quantified in terms of information transfer. We apply the approach to simulations of deterministic and stochastic systems, showing its superiority over standard uniform embedding. Effects of quantization, data length, and noise contamination are investigated. As practical applications, we consider the assessment of cardiovascular regulatory mechanisms from the analysis of heart period, arterial pressure, and respiration time series, and the investigation of the information flow across brain areas from multichannel scalp electroencephalographic recordings.
\end{abstract}

DOI: 10.1103/PhysRevE.83.051112

PACS number(s): 02.50.Sk, 05.45.Tp, 05.45.Xt, 87.19.lo

\section{INTRODUCTION}

Quantification of the direction and strength of the coupling among simultaneously observed systems from the analysis of time series recordings is an important topic currently under investigation in many fields of science. In the study of physiological systems, a huge number of theoretical and experimental studies have been published in recent years, with typical examples regarding cardiovascular and cardiorespiratory interactions [1], and synchronization of neural signals [2]. The mathematical formulation of the concept of causality given by Wiener [3] and formalized by Granger in the context of linear regression models of stochastic processes [4] has become a reference approach for identifying directional interactions between coupled systems. Even though its original formulation was based on measuring predictability improvements in bivariate linear autoregressive models, Granger causality was successfully extended to multivariate linear models [5], as well as to nonlinear systems [6]. While they have been proven useful in addressing specific issues of cardiovascular or neural interactions, model-based approaches are often of difficult generalization as they may suffer from the shortcomings of model mis-specification.

As an alternative to linear or nonlinear parametric models, information-theoretic methods [7] constitute a valid, modelfree approach to assess nonlinear causality for both deterministic and stochastic systems. The key for assessing causality within the information-theoretic framework is to incorporate the flow of time into the desired measure through the utilization of conditional probabilities. According to this concept, Porta et al. [8] exploited the definition of conditional entropy [9] to measure causality in bivariate systems as the amount of information carried by one process when the past of the

\footnotetext{
*Author to whom correspondence should be addressed: luca.faes@unitn.it
}

other process is known. Further developing this idea through independent approaches, Schreiber [10] and Palus et al. [11] defined the concepts of transfer entropy and conditional mutual information, which have been shown to be equivalent later on [12]. These measures allow us to quantify the amount of information exchanged between two systems separately for both directions and, when desired, conditional to common signals. The assessment of causality based on information transfer is framed in different terms with respect to the Granger approach, the first involving the concept of uncertainty and the second the concept of predictability. Nevertheless, the relation between transfer entropy and Granger causality is known $[11,13]$, and analytical equivalence has been very recently demonstrated [14], bridging information-theoretic approaches to the classical predictability-based approaches for the evaluation of causality.

Even though various measures have been proposed in the past [15], the practical application of information-theoretic concepts to the evaluation of Granger causality on multivariate experimental time series is not a trivial task. One major issue is related to how to perform proper conditioning, i.e., to choose which and how many past states of the considered processes have to be considered for the estimation of conditional entropy. This problem can be seen in terms of performing suitable multivariate embedding of the considered set of time series [16]. The large majority of approaches applied so far implicitly follow uniform multivariate embedding schemes where the components to be included in the embedding vectors are selected arbitrarily or separately for each time series $[15,17,18]$. The obvious arbitrariness and redundancy introduced by this strategy are likely to cause problems such as overfitting and detection of false causalities $[12,13]$. Another issue is related to the estimation of entropies themselves. While several estimators designed for multidimensional spaces can be applied for conditional entropy estimation [7], a common problem is the bias that increasingly affects 
conditional entropy estimates at augmenting dimensionality of the embedding vectors. These issues become critical when factors typical of practical applications, such as the data length and the signal-to-noise ratio, decrease to the values commonly encountered in experimental short-term time series analysis.

In this work, we focus on applicability of information theoretic methods for the evaluation of nonlinear Granger causality on multivariate deterministic and/or stochastic coupled processes. Taking inspiration from the ideas outlined in Refs. [16] and $[8,19]$, we propose an information-theoretic approach to assess causality among multiple time series measured from coupled dynamical systems, which integrates together a sequential procedure for nonuniform multivariate embedding for allowing proper conditioning, and a corrected estimate of the conditional entropy allowing bias compensation. The method is described in detail in Sec. II, tested on several simulations of deterministic and stochastic systems in Sec. III, and applied on physiological time series from cardiovascular and brain systems in Sec. IV. Summary results are discussed in Sec. V.

\section{METHODS}

\section{A. Assessment of nonlinear Granger causality through conditional entropy estimation}

Let us consider $M$ physical processes $X_{1}, \ldots, X_{M}$, as interacting subsystems of a whole observed stochastic or deterministic system. Given the $M$ time series of length $N,\left\{x_{m, n}\right\}, m=$ $1, \ldots, M, n=1, \ldots N$, generated from the processes, we treat them as short, and possibly noisy, descriptors of the states assumed by the subsystems over time; the state $x_{m, n}$ visited by $X_{m}$ at time $n$ is associated with the probability $p\left(x_{m, n}\right)$.

In order to describe the dynamics of one of the processes, say $X_{i}$, we need to define composite processes consisting of words, or embedding vectors. For instance, $X_{i}$ may be characterized through a uniform univariate embedding procedure whereby the $k$ past states of the process, collected in the embedding vector $\mathbf{x}_{i, n}^{(k)}=\left(x_{i, n-1}, \ldots, x_{i, n-k}\right)$ with joint probability $p\left(\mathbf{x}_{i, n}^{(k)}\right)$, are exploited to describe the current state $x_{i, n}$. The dynamical state of the process is reflected in the transition probabilities $p\left(x_{i, n} \mid \mathbf{x}_{i, n}^{(k)}\right)$, measuring the probability for $X_{i}$ to be in the state $x_{i, n}$ at time $n$ when the preceding states at times $n-1, \ldots, n-k$ are $x_{i, n-1}, \ldots, x_{i, n-k}$. The uncertainty of a transition into a new state is quantified through the conditional entropy [9]:

$$
H\left(x_{i, n} \mid \mathbf{x}_{i, n}^{(k)}\right)=-\sum_{n} p\left(x_{i, n}, \mathbf{x}_{i, n}^{(k)}\right) \ln p\left(x_{i, n} \mid \mathbf{x}_{i, n}^{(k)}\right)
$$

where the sum extends over all states visited by $X_{i}$. The conditional entropy may be expressed as $H\left(x_{i, n} \mid \mathbf{x}_{i, n}^{(k)}\right)=$ $H\left(x_{i, n}, \mathbf{x}_{i, n}^{(k)}\right)-H\left(\mathbf{x}_{i, n}^{(k)}\right)$, where $H(\cdot)$ denotes the entropy of a vector variable measuring the amount of information carried by the variable. As a result, the conditional entropy in Eq. (1) may be interpreted as the residual information carried by the present of the $i$ th process when its past is known up to a lag $k$.

According to the Granger notion of predictability improvement [4], causality from the process $X_{j}$ to the process $X_{i}$ is assessed comparing the entropy of $X_{i}$ conditioned on its own past and the past of all processes except $X_{j}$, and its entropy conditioned on the past of all processes, including $X_{j}$. The two steps require us to design procedures for multivariate embedding where components from different processes are included into the embedding vectors. In the classical uniform embedding framework, the embedding vectors are

$$
\begin{gathered}
\mathbf{x}^{\left(K_{j}\right)}=\left(\mathbf{x}_{1, n}^{\left(k_{1}\right)}, \ldots, \mathbf{x}_{j-1, n}^{\left(k_{j-1}\right)}, \mathbf{x}_{j+1, n}^{\left(k_{j+1}\right)}, \ldots, \mathbf{x}_{M, n}^{\left(k_{M}\right)}\right), \\
\mathbf{x}^{(K)}=\left(\mathbf{x}_{1, n}^{\left(k_{1}\right)}, \ldots, \mathbf{x}_{M, n}^{\left(k_{M}\right)}\right)
\end{gathered}
$$

with dimensions

$$
K_{j}=\sum_{\substack{m=1 \\ m \neq j}}^{M} k_{m}
$$

and $K=\sum_{m=1}^{M} k_{m}$, respectively. These vectors are used as conditioning vectors to calculate the entropies $H\left(x_{i, n} \mid \boldsymbol{x}^{(K j)}\right)$ and $H\left(x_{i, n} \mid \boldsymbol{x}^{(K)}\right)$, which are in turn combined to assess the resolution of uncertainty (i.e., increase of predictability or decrease of information) of the present of $X_{i}$ yielded by consideration of the past of $X_{j}$ :

$$
C_{j \rightarrow i}=1-\frac{H\left(x_{i, n} \mid \mathbf{x}^{(K)}\right)}{H\left(x_{i, n} \mid \mathbf{x}^{\left(K_{j}\right)}\right)} .
$$

The index $C_{j \rightarrow i}$ ranges between 0 and 1 , and its magnitude reflects the coupling strength as a measure of the amount of information carried by $X_{i}$ which is explained exclusively by the past of $X_{j}$. This quantity can be taken as a normalized version of the transfer entropy proposed by Schreiber [10] when more than two processes are considered.

The estimation of the quantity in Eq. (3) from multiple time series poses problems related to appropriateness of the embedding procedure. As a matter of fact, the traditional procedures for uniform embedding, yielding the embedding vectors in Eq. (2), introduce issues of arbitrariness and redundancy that may become critical in the assessment of causality. While in linear Granger causality appropriate order selection methods may be implemented [20], in nonlinear extensions like ours the selection of the embedding dimensions $k_{i}$, and of the relation between the overall dimensions $K_{j}$ and $K$, is not straightforward. In addition, even with a proper selection of the embedding dimension, the uniform scheme may include redundant terms that bring overlapping information, with possible consequences in the causality estimates. From a theoretical standpoint, in deterministic systems an exhaustive embedding based only on components from $X_{i}$ (with dimension $k \geqslant 2 d+1$, where $d$ is the dimension of the manifold where the observed motion develops [21]) would be sufficient for explaining the dynamics of $X_{i}$, and thus predicting its states; in this case, the index $C_{j \rightarrow i}$ would be indeterminate regardless of the strength of coupling exerted from $X_{j}$ to $X_{i}$. From a practical standpoint, an uncontrolled inclusion of components into the embedding vectors would easily lead to overfitting and detection of false causalities [13]. Another aspect is related to the known bias affecting estimation of the conditional entropy, which leads to entropy values becoming more and more underestimated at increasing the dimension of the embedding vectors $[8,19]$. The modifications to the traditional procedures for embedding formation and conditional entropy estimation proposed in the following section aim at addressing these critical issues. 


\section{B. Nonuniform multivariate embedding and corrected conditional entropy}

We propose to assess causality through a procedure for nonuniform multivariate embedding in which the form of the embedding vectors is not imposed a priori like in Eq. (2), but is determined in a sequential way selecting progressively the terms that contribute most to the description of the observed dynamics. These terms are taken from a set of candidate terms which includes the past states of the processes under analysis, $X_{1}, \ldots, X_{M}$. As reported above, to quantify causality from $X_{j}$ to $X_{i}$ we need to compare the entropy of $X_{i}$ measured either after conditioning on the past of all processes, or after conditioning on the past of all processes except $X_{j}$. To this end, two different sets of initial candidate terms are defined, respectively including and excluding the past states of $X_{j}: \Omega=\left\{x_{i, n-l} \mid i=1, \ldots, M ; l=1, \ldots, L\right\}$ and $\Omega^{-j}=$ $\left\{x_{i, n-l} \mid i=1, \ldots, M, i \neq j ; l=1, \ldots, L\right\}$ ( $L$ is the maximum lag at which the past of each process is investigated; it may vary across processes). Given the candidate set, the procedure starts with an empty embedding vector $\mathbf{x}^{(0)}$, and then proceeds as follows:

(i) for each $k \geqslant 1$, form the candidate vector $\left(x, \mathbf{x}^{(k-1)}\right)$ where $x$ is an element of the candidate set not already present in $\mathbf{x}^{(k-1)}$;

(ii) compute the entropy of $X_{i}$ given the considered candidate vector, $H\left[x_{i, n} \mid\left(x, \boldsymbol{x}^{(k-1)}\right)\right]$;

(iii) repeat steps (i) and (ii) for each $x$ belonging to the candidate set and not already selected, and then retain the candidate $\tilde{x}$, which minimizes the conditional entropy, i.e., set $\mathbf{x}^{(k)}=\left(\tilde{x}, \mathbf{x}^{(k-1)}\right)$ such that $\tilde{x}=\arg _{x} \min H\left(x_{i, n} \mid\left(x, \mathbf{x}^{(k-1)}\right)\right)$;

(iv) if a minimum of the conditional entropy is found, i.e., the condition $H\left(x_{i, n} \mid \mathbf{x}^{(k)}\right)>H\left(x_{i, n} \mid \mathbf{x}^{(k-1)}\right)$ holds, exit the algorithm; otherwise increase $k$ and go back to step (i).

After exiting the algorithm, the selected embedding vector is the one which minimizes the conditional entropy, i.e., $\mathbf{x}^{(K)}$ such that $K=k-1$. The corresponding entropy is taken as a measure of the amount of information carried by $X_{i}$, which is not explained by the set of candidate terms; taking either $\Omega$ or $\Omega^{-j}$ as the initial set of candidates, the entropies $H\left(x_{i, n} \mid \mathbf{x}^{(K)}\right)$ and $H\left(x_{i, n} \mid \mathbf{x}^{(K j)}\right)$ are computed and used as in Eq. (3) to quantify causality from $X_{j}$ to $X_{i}$. As it selects progressively the candidate that minimizes conditional entropy [at step (iii)] and the length of the embedding vector that again minimizes conditional entropy [at step (iv)], the procedure described above optimizes the embedding of the multivariate time series in order to better explain the dynamics of the considered process starting from the considered set of initial candidate terms. We note that the criterion for candidate selection is based on information reduction rather than on temporal ordering. Hence it may happen that a past term is selected before a more recent one; however, this does not affect the resulting measure, as joint probabilities are insensitive to the ordering of components within vector variables. Another observation is that the sequential procedure described here does not guarantee convergence to the absolute minimum of conditional entropy. However, it was preferred to an exhaustive exploration of all possible combinations of candidate terms, which would become computationally intractable still at low embedding dimensions.
To yield conditional entropy estimates, which do not decrease monotonically at increasing the length of the conditioning vector, but exhibit a well defined minimum, we followed the strategy proposed in $[8,19]$. This strategy is based first on performing uniform quantization of the observed time series to compute conditional entropy, and then on introducing a corrective term to compensate the bias occurring in entropy estimation. Each original time series $x_{m, n}$ is first normalized to have zero mean and unit variance, and then coarse grained spreading its dynamics over $Q$ quantization levels of amplitude $r=\left(x_{m}^{\max }-x_{m}^{\min }\right) / Q$, where $x_{m}^{\max }$ and $x_{m}^{\min }$ represent the minimum and maximum values of the normalized series. The resulting quantized series, denoted as $\xi_{m, n}$, take discrete values within the alphabet of $Q$ symbols $A=\{0,1, \ldots, Q-1\}$. As a result, uniform quantization applied to a given embedding vector $\mathbf{x}^{(k)}$ builds a uniform partition of the $k$-dimensional state space into $Q^{k}$ disjoint hypercubes of size $r$; all vectors falling within the same hypercube are associated with the same quantized vector $\xi^{(k)}$ and are thus indistinguishable within the tolerance $r$. The entropy of $\mathbf{x}^{(k)}$ is approximated with the entropy of its discrete version $\boldsymbol{\xi}^{(k)}: H\left(\boldsymbol{\xi}^{(k)}\right)=-\sum_{\xi^{(k)} \in \mathrm{A}^{k}} p\left(\boldsymbol{\xi}^{(k)}\right) \ln p\left(\boldsymbol{\xi}^{(k)}\right)$, where $p\left(\xi^{(k)}\right)$ is an estimate of the joint probability $p\left(\mathbf{x}^{(k)}\right)$ obtained as the frequency of occurrence of $\boldsymbol{\xi}^{(k)}$ within $A^{k}$. Then, an estimate of the conditional entropy $H\left(x_{i, n} \mid \mathbf{x}^{(k)}\right)$ is given by

$$
H\left(\xi_{i . n} \mid \boldsymbol{\xi}^{(k)}\right)=H\left(\xi_{i . n}, \boldsymbol{\xi}^{(k)}\right)-H\left(\boldsymbol{\xi}^{(k)}\right) .
$$

As pointed out in $[8,19]$, when the conditional entropy is estimated from time series of limited length, it always decreases towards zero with increasing the embedding dimension $k$. This effect results from the fact that, letting $k$ increase, an increasing number of vectors $\mathbf{x}^{(k)}$ will be found alone within a hypercube of the $k$-dimensional space and, as a consequence, also the vectors $\left(x_{i, n}, \mathbf{x}^{(k)}\right)$ will be alone in the $(k+1)$-dimensional space. Therefore their contribution to $H\left(x_{i, n} \mid \mathbf{x}^{(k)}\right)$ is null, and a bias towards a reduction of the entropy rate is introduced. To counteract this bias, we use the following corrected conditional entropy measure:

$$
H^{c}\left(\xi_{i, n} \mid \xi^{(k)}\right)=H\left(\xi_{i, n} \mid \xi^{(k)}\right)+n\left(\xi^{(k)}\right) H\left(\xi_{i, n}\right),
$$

where $n\left(\boldsymbol{\xi}^{(k)}\right)$ is the fraction of $k$-dimensional quantized vectors found only once in $A^{k}\left[0 \leqslant n\left(\boldsymbol{\xi}^{(k)}\right) \leqslant 1\right]$. With this correction, in the presence of a single point inside a hypercube, its null contribution is substituted with the maximal amount of information carried by a white noise with the same marginal distribution of the observed process $X_{i}$ [i.e., $H\left(\xi_{i, n}\right)$ ].

The application of the procedure for nonuniform embedding, with utilization of the corrected conditional entropy estimator described above, leads to the conditional entropy estimates $H^{c}\left(\xi_{i, n} \mid \xi^{(K)}\right)$ and $H^{c}\left(\xi_{i, n} \mid \xi^{(K j)}\right)$, respectively, when $\Omega$ and $\Omega^{-j}$ are taken as the initial set of candidate terms. These measures are combined as in Eq. (3) to obtain an estimate of the causal coupling from $X_{j}$ to $X_{i}$ :

$$
C_{j \rightarrow i}^{c}=1-\frac{H^{c}\left(\xi_{i, n} \mid \xi^{(K)}\right)}{H^{c}\left(\xi_{i, n} \mid \xi^{\left(K_{j}\right)}\right)} .
$$




\section{SIMULATED SYSTEMS}

This section reports the evaluation of the proposed approach on numerical examples reproducing different conditions of interaction among multiple subsystems. We consider both nonlinear deterministic and linear stochastic simulated systems, with varying number of interacting processes and coupling degrees. The effect of relevant factors such as time series length, level of noise contamination, and number of quantization levels used for entropy estimation are investigated.

In order to perform reliable detection of causality from one process to another, we use a statistical approach for the detection of the significance of each specific estimated value of causal coupling. The approach performs a comparison between the original value of the considered measure and the distribution of such a measure under the null hypothesis of absence of coupling over the assigned causal direction. As the analytic null distribution of the proposed causality measure is not available, we exploit a method based on surrogate data to reproduce it empirically. First, we use the technique of time shifted surrogates $[16,18,22]$ to generate multiple $(S=$ 40 in this study) realizations of the input process $X_{j}$, which share the statistical structure of the original time series $x_{j, n}$, but are not causally coupled to the output time series $x_{i, n}$. The technique simply shifts the original series $\left(x_{j, 1}, \ldots, x_{j, n}\right)$ of a randomly selected lag $l(l>20)$ to yield the surrogate series $\left(x_{j, l+1}, \ldots, x_{j, N}, x_{j, 1, \ldots, x_{j, l}}\right)$. The causal coupling from $X_{j}$ to $X_{i}$ is then computed both for the set of original time series $\left\{x_{1, n}, \ldots, x_{M, n}\right\}$ and for the $S$ sets of series in which $x_{j, n}$ is substituted by one of the surrogate series. As the null distribution of the causal coupling is hardly symmetric, we perform the test for significance on the basis of rank ordering. According to the distribution-free plotting position formula devised in [23], we estimate the type-I error probability as $\alpha=1-(i-0.326) /(S+1+0.348)$, where $i$ is the position taken by the original causal coupling within the ascending ordered sequence of original and surrogate causal coupling values (the test is one-sided); for instance, if the statistic for the original series is the second largest $(i=40)$, the null hypothesis of absence of causality can be rejected with significance $\alpha=0.0405$. We remark that the significance levels set in this way are appropriate for testing causality over the assigned selected direction, while a correction for multiple comparisons would be necessary if one had to test for the presence of causality within the whole observed system (according to the null hypothesis of absence of coupling in at least one direction).

\section{A. Coupled Rössler-Lorenz system}

The first considered example is the unidirectionally coupled Rössler-Lorenz map (with $M=2$ subsystems) [24], where an autonomous Rössler system,

$$
\begin{gathered}
\dot{z}_{1}(t)=-6\left[z_{2}(t)+z_{3}(t)\right], \\
\dot{z}_{2}(t)=6\left[z_{1}(t)+a z_{2}(t)\right], \\
\dot{z}_{3}(t)=6\left[b+z_{1}(t) z_{3}(t)-d z_{3}(t)\right],
\end{gathered}
$$

with parameters $a=0.2, b=0.2, d=5.7$, drives a Lorenz system, in which the equation for the variable $y_{2}$ is augmented by a driving term involving the variable $z_{2}$ with coupling parameter $C$ :

$$
\begin{gathered}
\dot{y}_{1}(t)=10\left[-y_{1}(t)+y_{2}(t)\right], \\
\dot{y}_{2}(t)=28 y_{1}(t)-y_{2}(t)-y_{1}(t) y_{3}(t)+C z_{2}^{2}(t), \\
\dot{y}_{3}(t)=y_{1}(t) y_{2}(t)-(8 / 3) y_{3}(t) .
\end{gathered}
$$

We denote the Rössler and Lorenz systems as $X_{1}$ and $X_{2}$, and consider $z_{2}$ and $y_{2}$ as driving and driven variables, which we denote as $x_{1}$ and $x_{2}$, respectively. The differential equations were iterated using an explicit Runge-Kutta method with a time step of 0.01. After discarding the first $10^{5}$ iterations to eliminate transients, time series $x_{1, n}$ and $x_{2, n}$ of length $N=$ 10000 were generated. The coupling strength was varied in the range $C=\{0,0.5,1,1.5,2,2.5,3\}$; for each value of $C, 100$ realizations of the processes were generated varying the initial conditions for the two subsystems. Moreover, to investigate dependence of the results on data size, the analysis was repeated for shortened versions of the time series obtained cutting the original series to the reduced lengths $N=100,300$, $500,750,1000$, or 5000 .

A representative example of the analysis is reported in Fig. 1. The sequential procedure for nonuniform embedding applied to the Lorenz system starting from a set of initial candidates excluding terms from the Rössler system [Fig. 1(a); $x_{2, n}$ is described from $\left.\Omega^{-1}=\left\{x_{2, n-1}, \ldots, x_{2, n-10}\right\}\right]$ terminates at the step $K_{1}=3$, yielding the embedding vector $\mathbf{x}^{(K 1)}=$ $\left(x_{2, n-1}, x_{2, n-2}, x_{2, n-8}\right)$ and the corresponding minimum corrected conditional entropy $H_{c}\left(\xi_{2, n \mid} \xi^{(K 1)}\right)=0.708$. When Rössler terms are included in the set of initial candidates [Fig. 1(b), $\left.\Omega=\left\{x_{1, n-1}, \ldots, x_{1, n-10}, x_{2, n-1}, \ldots, x_{2, n-10}\right\}\right]$, the procedure selects the same terms for the first two steps, but includes $x_{1, n-6}$ in place of $x_{2, n-8}$ at the third step; this leads to a reduction in the minimum corrected conditional entropy, which is now $H^{c}\left(\xi_{2, n} \mid \xi^{(K)}\right)=0.653$, and consequently to a positive causal coupling $C_{1 \rightarrow 2}^{c}=0.078$. The significance of this value is assessed, repeating the analysis after substitution of the input series $x_{1, n}$ with a set of time-shifted surrogates. This alters the procedure executed with $\Omega$ as the initial candidate set: as shown in Fig. 1(b), the minimum corrected conditional entropy values estimated for the surrogates are always higher than that estimated for the original series, so that the original causal coupling is the largest of the sequence of original and surrogate causal coupling values, and the test is rejected with significance $\alpha=0.0163$. When the coupling over the opposite causal direction (from Lorenz to Rössler) is investigated, exclusion or inclusion of terms of the Lorenz system $X_{2}$ from the set of initial candidates used to describe the Rössler system $X_{1}$ does not alter the procedure. Indeed, the selected embedding vectors in Figs. 1(c) and Fig. 1(d) are the same, $\mathbf{x}^{(K 2)}=\mathbf{x}^{(K)}=\left(x_{1, n-5}, x_{1, n-6}, x_{1, n-1}, x_{1, n-4}\right)$; as a consequence, the minimum corrected conditional entropy does not change and $C_{2 \rightarrow 1}^{c}=0$. The same embedding vector is found also for the surrogate time series [all lines overlap in Fig. 1(d)], and thus the coupling is not significant over this direction. 
(a)

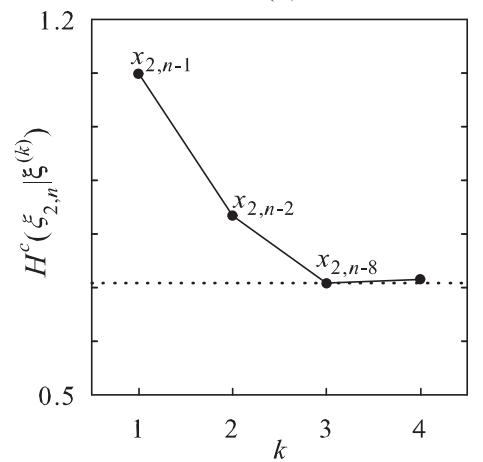

(c)

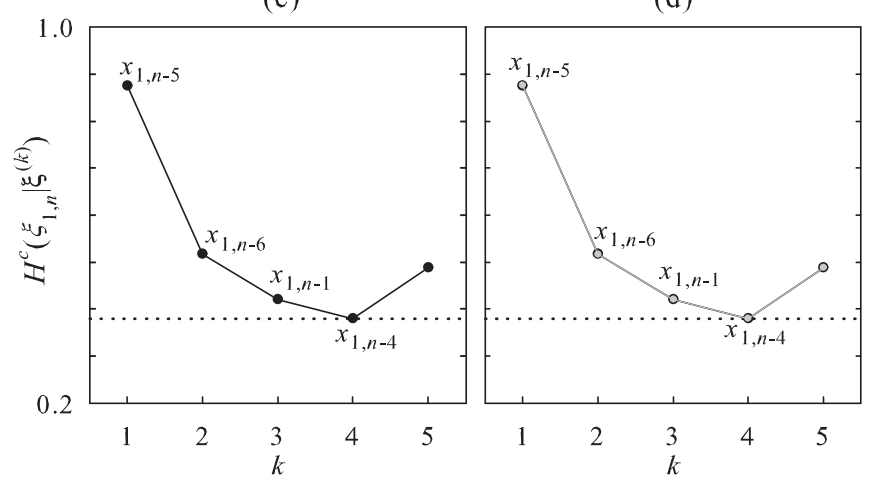

FIG. 1. Example of application of the sequential procedure for nonuniform multivariate embedding for the simulation with a Rössler system $\left(X_{1}\right)$ driving a Lorenz system $\left(X_{2}\right)$. Plots depict the corrected entropy conditioned to the optimal candidate vector $\mathbf{x}^{(k)}$, estimated at each step $k$ of the procedure, for $X_{2}$ when the set of initial candidate terms is $\Omega^{-1}$ (a) or $\Omega$ (b), and for $X_{1}$ when the set is $\Omega^{-2}$ (c) or $\Omega$ (d). The candidate term selected at each step is indicated in the plot, and the horizontal dotted line indicates the minimum estimated corrected conditional entropy. Multiple lines plotted in gray represent results of the analysis performed on different sets of surrogate time series. Analysis parameters: coupling strength $C=1.5$, time series length $N=500$, number of quantization levels $Q=6$, maximum lag of the terms included in the initial sets of candidates $L=10$.

Figure 2 summarizes the results of the analysis performed at varying the coupling strength $C$. As imposed by the procedure, the embedding vectors contain only terms of the studied system when terms of the other system are excluded from the set of initial candidates [in Figs. 2(a) and 2(d), all terms are from $X_{2}$ and from $X_{1}$, respectively]. When the procedure is repeated starting from the most comprehensive set of initial candidate terms $\left(\Omega=\left\{x_{1, n-1}, \ldots, x_{1, n-10}, x_{2, n-1}, \ldots, x_{2, n-10}\right\}\right)$, terms from the input system may enter the embedding vector describing the output system. Specifically we note that, increasing the coupling parameter $C$, an increasing number of terms from $X_{1}$ enter the embedding vector describing $X_{2}$ [Fig. 2(b)]; this greater importance of the input terms is reflected by increasing values of the causal coupling from $X_{1}$ to $X_{2}$ [Fig. 2(c), black squares]. On the contrary, the embedding vector describing $X_{1}$ keeps containing exclusively terms from $X_{1}$ even when terms from $X_{2}$ could be selected [Fig. 2(e)]; as a consequence, no variations in the minimum corrected conditional entropy are observed and the causal coupling from $X_{2}$ to $X_{1}$ is uniformly zero [Fig. 2(f)]. A slight exception to this behavior occurs for the largest values of the coupling parameter, when a small percentage of realizations contain one term from $X_{2}$ [Fig. 2(e) with $C=2.5$ and $C=3$ ] and this results in slightly positive values of $C_{2 \rightarrow 1}^{c}$ [Fig. 2(f)]. A possible explanation of this trend is in the fact that for $C>2$ the systems tend to approach a state of generalized synchronization [25] in which $\Omega^{-1}$ and $\Omega^{-2}$ contain common information; in this condition, behaviors such as the increase of coupling over the uncoupled direction, or the decrease of coupling over the coupled direction, observed in Ref. [16], are likely to occur. The dimension of the embedding vector is estimated around 4 for both systems and both sets of initial candidate components. This value is larger than the true embedding dimension, which is known to be 3 for individual uncoupled Rössler and Lorenz systems. Slight variations in the number of components selected as a function of $C$ are observed for $X_{2}$, reflecting the fact that the presence of a driving term may alter the dimension of the system. On the contrary, both $K_{2}$ and $K$ do not vary with $C$ for the system $X_{1}$, correctly indicating that the dynamics of the driving system is not affected by the coupling strength.

Figure 2(c) reports also the causal coupling evaluated when a uniform embedding procedure, implemented selecting the terms through progressive increase of the lags and alternation between the two series, was used to estimate the causal coupling (white squares). The comparison with the proposed nonuniform embedding evidences the inability of the uniform embedding to detect coupling below $C=2$, and the weaker coupling detected for $C \geqslant 2$.

With the parameter setting of Eq. (7a), which corresponds to that commonly chosen for studying directional coupling in the Rössler-Lorenz system [16,24], the driving Rössler system is in a phase-coherent regime exhibiting a chaotic attractor with a relatively simple topology. To investigate coupling detection in the presence of a more complex topological structure, we set $a=0.25, b=0.1, d=-8.5$ in Eq. (7a), so that the Rössler attractor becomes a funnel attractor [26]. The results reported in Figs. 2(c) and 2(f) show that the causal coupling (black circles) was detected also in this case with the driving system in the funnel chaotic regime, often even with a better performance (larger coupling detected) than during the phasecoherent regime. Hence it appears that the evolution of the driving system over more complex chaotic manifolds is not detrimental for the detection of nonlinear Granger causality towards the driven system.

The dependence of the procedure on the length of the considered time series is analyzed in Fig. 3. As seen in Fig. 3(a), the procedure yields a causal coupling from the Rössler to the Lorenz system increasing progressively with the coupling parameter $C$ even for time series as short as $N=$ 100 samples. The values of causal coupling tend, with some exceptions, to be higher for longer time series. Moreover, the procedure is able to detect the unidirectional nature of the coupling for all time series length, as coupling on the opposite direction remains very low [Fig. 3(b)]. Figures 3(c) and 3(d) report the estimated number of realizations (out of 100) in which the causal coupling was detected as significant according to the adopted statistical test (the criterion adopted here takes as significant up to the third largest coupling, corresponding to a significance level $\alpha=0.0647$ ). The plot in Fig. 3(c) reflects that of the causal coupling, with a 
(a)

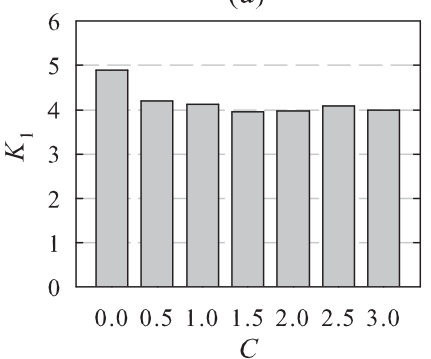

(d)

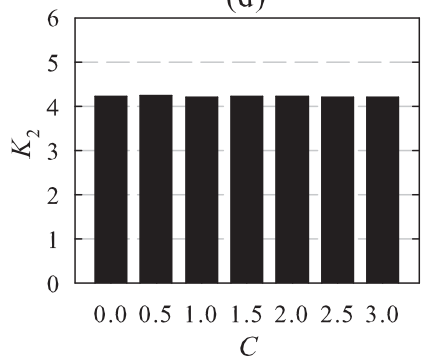

(b)

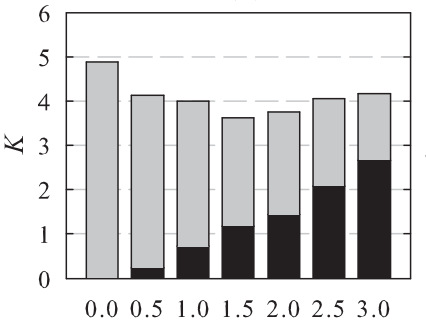

C

(e)

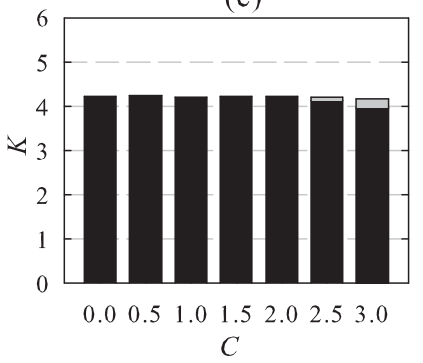

(c)
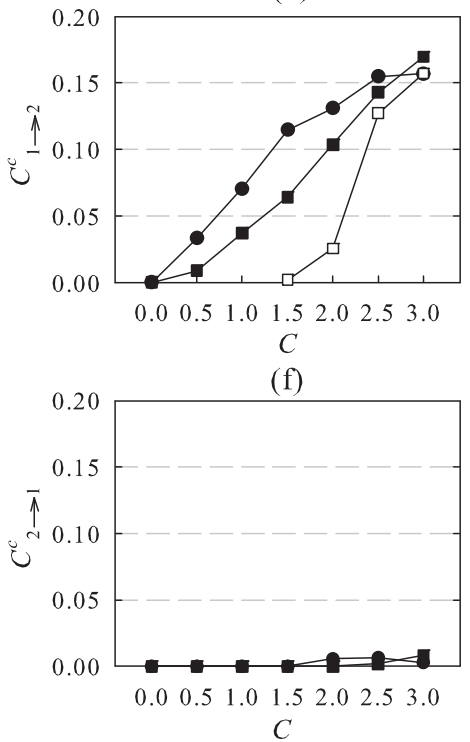

FIG. 2. Composition of the embedding vectors and corresponding causal coupling yielded for the simulation with a Rössler system $\left(X_{1}\right)$ in the phase-coherent regime driving a Lorenz system $\left(X_{2}\right)$. Left and middle panels depict the dimension of the embedding vectors for the description of $X_{2}$ when the set of initial candidate terms is $\Omega^{-1}$ (a) or $\Omega$ (b), and for the description of $X_{1}$ when the set of initial candidate terms is $\Omega^{-2}$ (d) or $\Omega$ (e). Each bar is partitioned with two colors indicating the number of terms from $X_{1}$ (black) and from $X_{2}$ (gray) forming the embedding vector. Right panels depict the corresponding causal coupling estimated from $X_{1}$ to $X_{2}$ (c) and from $X_{2}$ to $X_{1}$ (f) (black squares). Panel (c) includes also coupling values obtained by uniform embedding (white squares), while panels (c) and (f) include coupling values obtained for the parameter setting of a funnel regime for the Rössler system (black circles). All values in the plots are the average over 100 realizations of the simulation and are expressed as a function of the coupling strength $C$. Analysis parameters: $N=500, Q=6, L=10$.

percentage of rejection of the null hypothesis of uncoupling which increases with the imposed coupling strength and with the time series length. While the coupling is hardly detected for $C=0.5\left(n_{1 \rightarrow 2}\right.$ is $\sim 50 \%$ for $N=1000$ and lower for shorter series), the percentage of detection is substantial (>80\%), e.g., for $\{C \geqslant 1, N \geqslant 750\},\{C \geqslant 1.5, N \geqslant 500\}$, and $\{C \geqslant 2, N \geqslant 300\}$. As perceived in Fig. 2, Figs. 3(b) and 3(d) show that the coupling over the uncoupled direction starts to be nonzero and significant for $C \geqslant 2.5$. Again, this result may be ascribed rather than to the presence of spurious causality to the emergence of generalized synchronization, which favors the detection of bidirectional interactions [25].

\section{B. Multivariate coupled Henon maps}

As a second example, we consider $M=3$ discrete-time deterministic systems interacting in accordance with the equations

$$
\begin{gathered}
x_{1, n}=1.4-x_{1, n-1}^{2}+0.3 x_{1, n-2}+0.08\left(x_{1, n-1}^{2}-x_{2, n-1}^{2}\right), \\
x_{2, n}=1.4-x_{2, n-1}^{2}+0.3 x_{2, n-2}+0.08\left(x_{2, n-1}^{2}-x_{1, n-1}^{2}\right), \\
x_{3, n}=1.4-\left[C x_{1, n-1}+(1-C) x_{3, n-1}\right] x_{3, n-1}+0.1 x_{2, n-2} .
\end{gathered}
$$

The time series $x_{1, n}$ and $x_{2, n}$ describe two bidirectionally coupled Henon systems [27] $X_{1}$ and $X_{2}$ with identical coupling strength of 0.08 , while the time series $x_{3, n}$ describes a Henon system $X_{3}$, which is driven both by $X_{1}$, through the coupling parameter $C$, and by $X_{2}$. After setting the value of $C$ in the range 0 to 1 , step $0.1,100$ realizations of Eq. (8) were generated varying the initial conditions and discarding the first $10^{5}$ iterations as transients. To study the deterioration of the method performance in the presence of noise, we performed the analysis for the clean time series $(N=300)$ and after contamination with additive white noise; the noise level was varied to obtain a signal-to-noise ratio of $25,20,15$, and $10 \mathrm{~dB}$.

Figure 4 depicts the results obtained, for the clean time series, when the procedure is performed to quantify causality from $X_{1}$ to $X_{3}$ at varying the coupling parameter $C$. As shown in Figs. 4(a) and 4(b), the dimension of the embedding vectors is very close to 3 for all values of $C$. While for $C>0$ this is the correct embedding dimension derived from the third equation in Eq. (8), for $C=0$ the procedure selects one term from $X_{2}$ and two terms from $X_{3}$ for the description of $X_{3}$ [Fig. 4(a)], thus overestimating the expected dimension which is known to be 2 . However, the selection of one term in excess from $X_{3}$ does not affect the evaluation of causality, since the same embedding vectors are selected also when terms of $X_{1}$ are included in the initial set of candidates [with $C=0$, Fig. 4(b) indicates the same composition of the embedding vectors as Fig. 4(a)] and, as a consequence, the causal coupling from $X_{1}$ to $X_{3}$ is equal to zero [Fig. 4(c)]. With $C>0$, the composition of the embedding vectors always varies when candidate terms from $X_{1}$ are allowed in the procedure (i.e., moving from $\Omega^{-1}$ to $\Omega$ as the initial set of candidates). Specifically, while starting from the set $\Omega^{-1}$ no terms from $X_{1}$ are selected by construction [Fig. 4(a)]; starting from the set $\Omega$ a given percentage of realizations contain a term from $X_{1}$ [black part of the bars in Fig. 4(b)]. Such an inclusion determines a deeper minimum in 

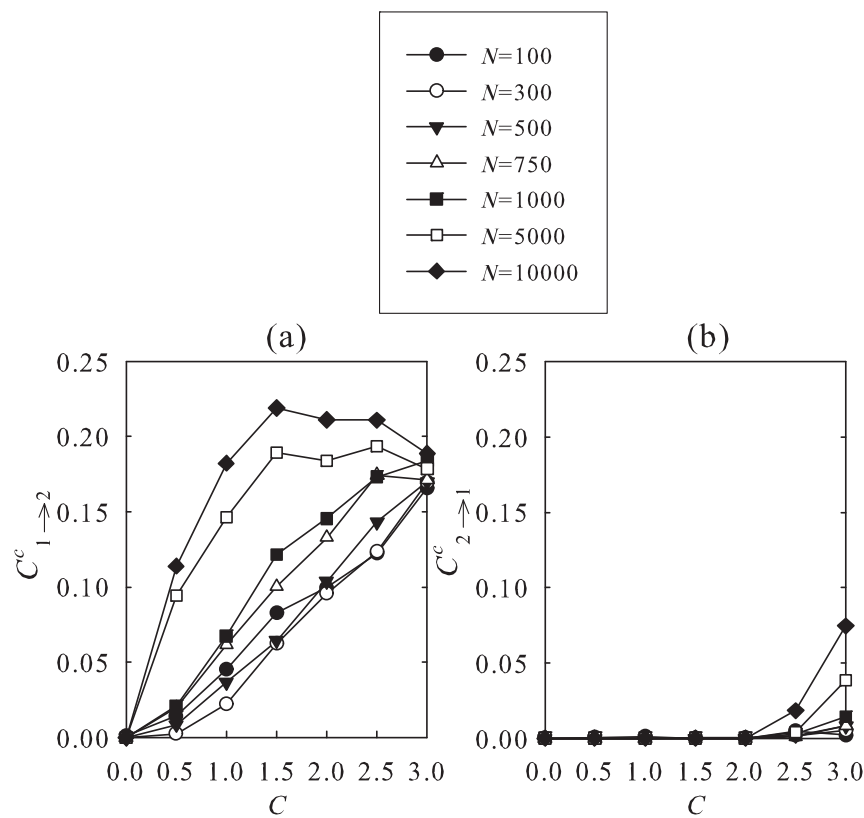

(c)

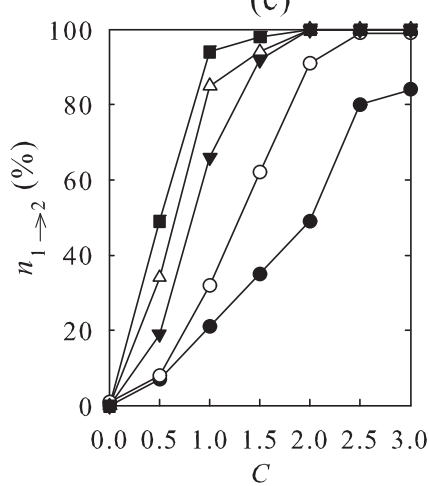

(d)

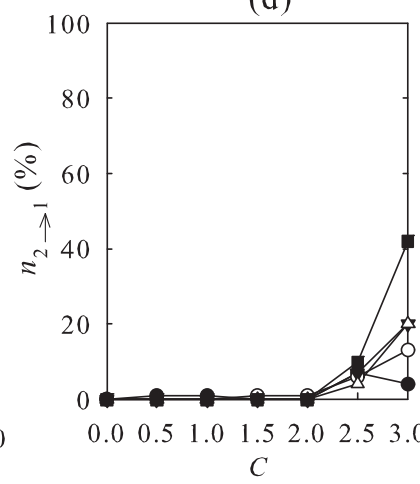

FIG. 3. Dependence on time series length for the simulation with a Rössler system $\left(X_{1}\right)$ driving a Lorenz system $\left(X_{2}\right)$. Plots depict the average causal coupling over 100 realizations estimated from $X_{1}$ to $X_{2}$ (a) and from $X_{2}$ to $X_{1}$ (b), and the percentage of realizations of the simulation for which the two causal couplings were significant according to the test based on surrogate data (c),(d), expressed as a function of the coupling parameter $C$ for different values of the series length $N$. Analysis parameters: $Q=6, L=10$.

the estimated corrected conditional entropy and thus a positive value for the causal coupling index [Fig. 4(c), black squares]. While the percentage of realizations in which an input term is selected is very small for $C=0.1$ (and in this case also the causal coupling is very low), it grows for higher imposed coupling strengths; for $C \geqslant 0.3$, all realizations contain at least one input term and the estimated causal coupling takes significant positive values. When $C$ approaches 1 , the composition of the embedding vectors resembles the imposed one (i.e., one term from each of the three systems is selected) and the corresponding causal coupling estimates stabilize at $\sim 0.24$. The comparison with the coupling index estimated through uniform embedding [Fig. 4(c), white squares] indicates the better ability of the proposed nonuniform embedding to detect the causality relation imposed in the simulated scheme. (a)

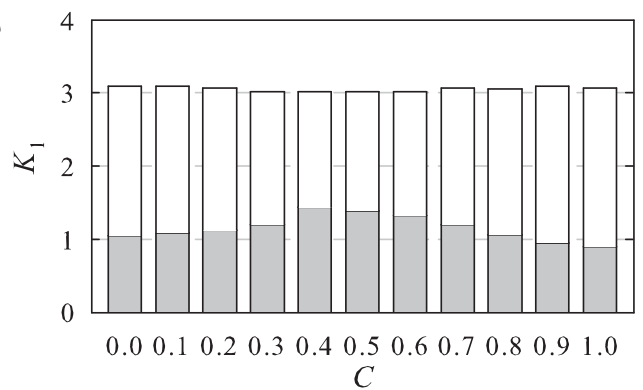

(b)
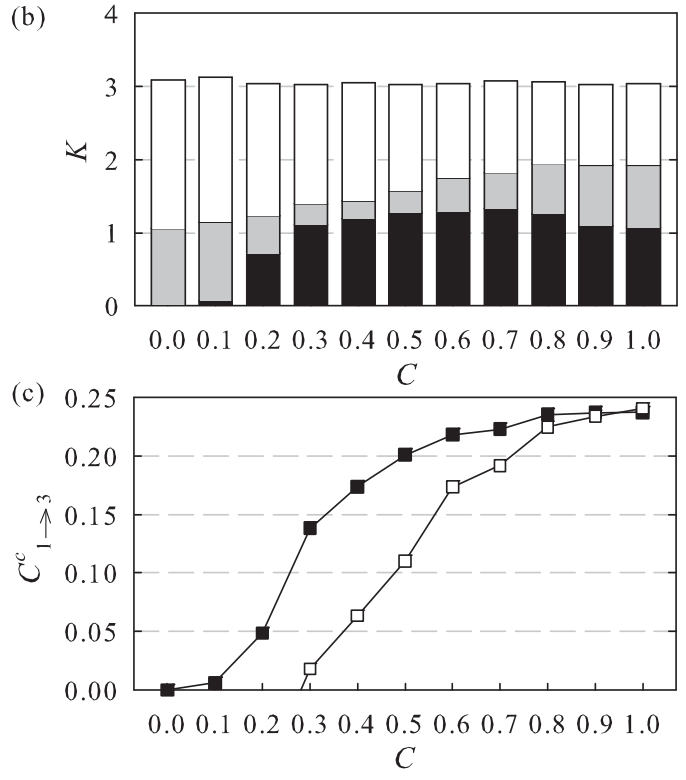

FIG. 4. Composition of the embedding vectors and corresponding causal coupling yielded for the simulation with three coupled Henon systems. Upper and middle panels depict the dimension of the embedding vectors for the description of $X_{3}$ when the set of initial candidate terms is $\Omega^{-1}$ (a) or $\Omega$ (b). Each bar is partitioned with three colors indicating the number of terms from $X_{1}$ (black), from $X_{2}$ (gray) and from $X_{3}$ (white) forming the embedding vector. The corresponding causal coupling estimated from $X_{1}$ to $X_{3}$ is in panel (c) (black squares), which includes also coupling values obtained by uniform embedding (white squares). All values in the plots are the average over 100 realizations of the simulation, and are expressed as a function of the coupling parameter $C$. Analysis parameters: $N=$ $300, Q=6, L=5$.

Figure 5 illustrates the effects of observational noise on the procedure for nonuniform embedding and causal coupling estimation. As clearly seen in Fig. 5(a), increasing levels of noise lead to decreasing values of the estimated causal coupling from $X_{1}$ to $X_{3}$. For all noise values except $10 \mathrm{~dB}$, this decrease seems not problematic, as the percentage of realizations in which the causal coupling was detected as significant [Fig. 5(b), imposed significance of the test $\alpha=$ $0.0647]$ remains zero for uncoupled dynamics $(C=0)$ and follows with good approximation the values obtained for the clean data for coupled dynamics $(C>0)$. With this series length, results appear deteriorated for a signal-to-noise ratio of $10 \mathrm{~dB}$, as a probability of false rejection of $8 \%$ is revealed with $C=0$ and the percentage of coupling detection does not rise above $80 \%$ for any value of $C>0$. 


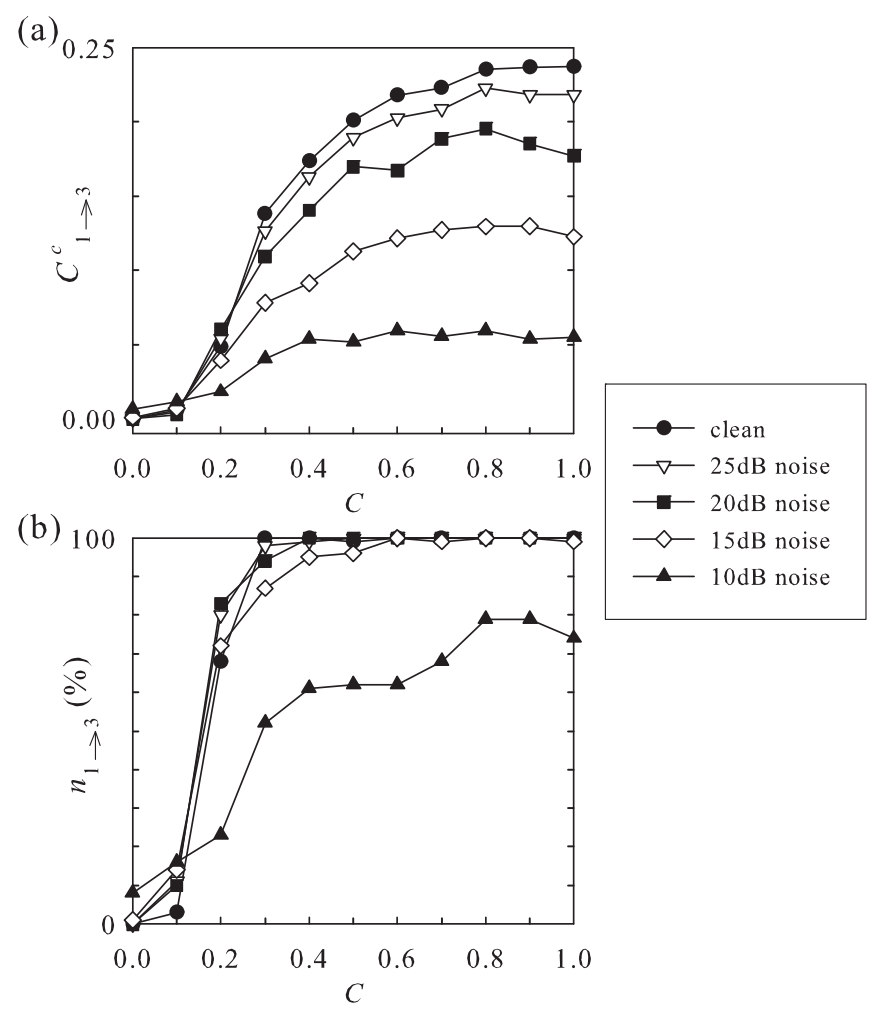

FIG. 5. Dependence on noise contamination for the simulation with three coupled Henon systems. Plots depict the average causal coupling over 100 realizations estimated from $X_{1}$ to $X_{3}$ (a), and the percentage of realizations of the simulation for which the causal coupling was significant according to the test based on surrogate data (b), expressed as a function of the coupling parameter $C$ for different levels of additive noise. Analysis parameters: $N=500, Q=6$, $L=5$.

\section{Coupled map lattice}

The third example consists of a ring lattice of $M=10$ unidirectionally coupled tent maps $[10,17]$ :

$$
\begin{gathered}
x_{m, n+1}=f\left[C x_{m-1, n}+(1-C) x_{m, n}\right], \\
m=1, \ldots, M\left(x_{0}=x_{M}\right),
\end{gathered}
$$

with $f(x)=1-2|0.5-x|$. The strength of the unidirectional coupling between each pair of adjacent maps of the lattice is varied from $C=0$ to $C=0.5$, step 0.05 . Figure 6 (circle symbols) shows the causal coupling along the directions $x_{m-1} \rightarrow x_{m}, x_{m} \rightarrow x_{m-1}$, and $x_{m-2} \rightarrow x_{m}$, averaged for each possible value of $m$. As expected, $C_{m-1 \rightarrow m}^{c}$ is positive and increases with the coupling strength [Fig. 6(a)], whereas $C_{m \rightarrow m-1}^{c}$ is uniformly zero regardless of the coupling strength [Fig. 6(b)], thus detecting the unidirectional nature of the coupling. Note that the coupling is also absent over the direction $x_{m-2} \rightarrow x_{m}$ [Fig. 6(c)], reflecting the fact that the proposed multivariate approach measures exclusively direct coupling between two sites of the lattice.

The comparison with a bivariate approach (Fig. 6, square symbols), in which the same analysis is performed considering only the two time series identifying the direction of interaction, shows that spurious coupling from $x_{m-2}$ to $x_{m}$ may be detected as a consequence of the indirect effect involving the series
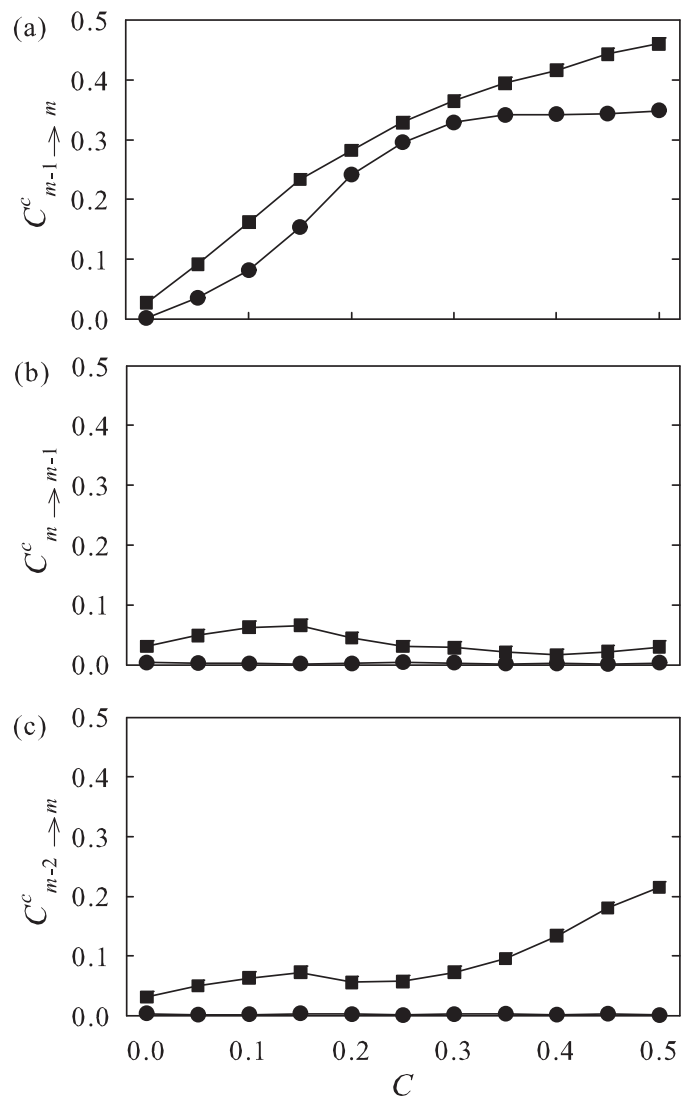

FIG. 6. Causal coupling estimated by means of a multivariate approach (circles) and a bivariate approach (squares) for the simulation of a coupled map lattice along the directions $x_{m-1} \rightarrow x_{m}$ (a), $x_{m} \rightarrow x_{m-1}$ (b), and $x_{m-2} \rightarrow x_{m}$ (c), averaged over 20 simulation runs, starting from random initial conditions and discarding $10^{4}$ points as transients. Analysis parameters: $N=300, Q=6, L=5$.

$x_{m-1}$ [Fig. 6(c)]. Moreover, for low values of $C$ the bivariate approach may not be able to detect the unidirectional coupling between adjacent sites, as some degree of information transfer is measured also over the uncoupled direction [Fig. 6(b)]. These results show how the utilization of a multivariate approach using the all of the information coming from highdimensional systems may be recommended to rule out spurious causal effects due to latent variables.

\section{Multivariate coupled stochastic processes}

The fourth example involves $M=4$ linear stochastic systems generated with the equations

$$
\begin{gathered}
x_{1, n}=2 \rho_{1} \cos \varphi_{1} x_{1, n-1}-\rho_{1}^{2} x_{1, n-2}+w_{1, n}, \\
x_{2, n}=0.5 x_{1, n-1}+0.5 x_{4, n-1}+w_{2, n}, \\
x_{3, n}=2 \rho_{3} \cos \varphi_{3} x_{3, n-1}-\rho_{3}^{2} x_{3, n-2}-0.5 x_{1, n-2}+w_{3, n}, \\
x_{4, n}=2 \rho_{4} \cos \varphi_{4} x_{4, n-1}-\rho_{4}^{2} x_{4, n-2}+x_{1, n-2}+w_{4, n},
\end{gathered}
$$

where $\rho_{1}=0.95, \rho_{3}=0.8, \rho_{4}=0.9, \varphi_{1}=0.628, \varphi_{3}=$ $1.256, \varphi_{4}=1.884$, and $w_{i, n}$ are Gaussian white noises with zero mean and unit variance. The processes $X_{1}, X_{3}$, and $X_{4}$ exhibit autonomous stochastic oscillations generated through the autoregressive terms: the strength and frequency of the 

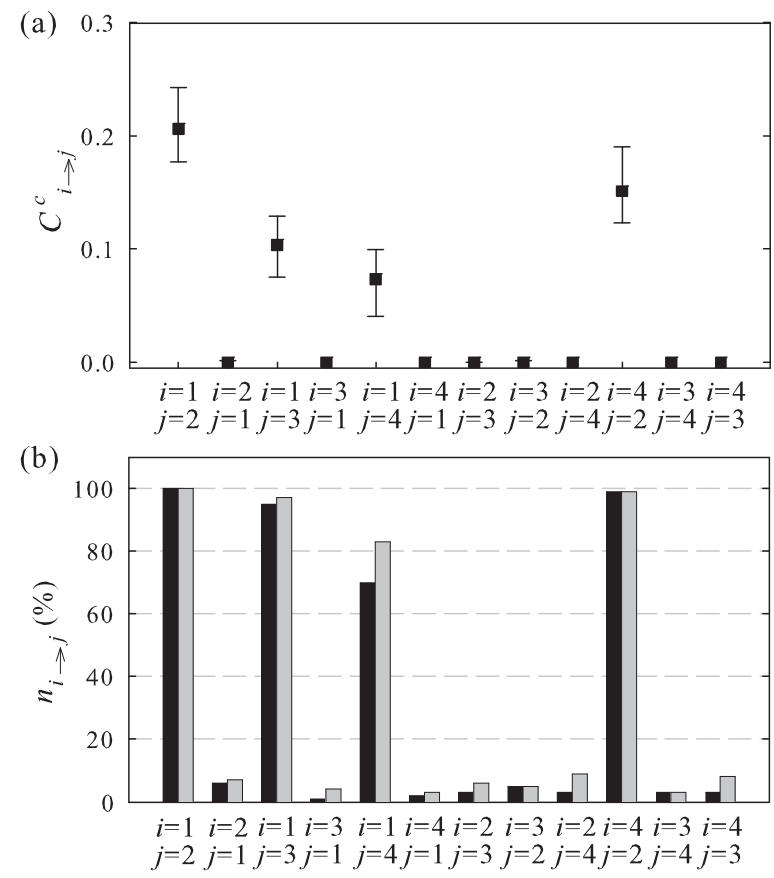

FIG. 7. Causal coupling strength and significance assessed for the simulation with four coupled stochastic systems. Plots depict, for each pair of processes $X_{i}$ and $X_{j}(i, j=1, \ldots, 4)$, the interquartile range (25th percentile, median and 75 th percentile) of the distribution of causal coupling from $X_{i}$ to $X_{j}$ estimated from 100 simulation runs (a), and the percentage of runs for which the coupling was significant according to the test based on surrogate data (b; black bars: significance $\alpha=0.0163$; gray bars: significance $\alpha=0.0647$ ). Analysis parameters: $N=300, Q=6, L=10$.

oscillation of the series $x_{i, n}$ are controlled respectively by the parameters $\rho_{i}$ and $\varphi_{i}$ included in the weights of the terms $x_{i, n-1}$ and $x_{i, n-2}(i=1,3,4)$. Directional connections at lag $k$ are then obtained from the process $X_{j}$ to the process $X_{i}$ setting a nonzero weight for the term $x_{j, n-k}$ in the right-hand side of the equation having $x_{i, n}$ as the left-hand side.

The results summarized in Fig. 7 indicate that the procedure is able to detect the causal relationship imposed in Eq. (10) and at the same time avoid the detection of spurious causality over the uncoupled directions. In fact, the distribution of causal coupling estimated over the 100 realizations is clearly larger than zero from $X_{1}$ to $X_{2}$, to $X_{3}$ and to $X_{4}$, and from $X_{4}$ to $X_{2}$, while the coupling is substantially absent over the remaining causal directions [Fig. 7(a)]. This behavior is reflected by the percentage of rejection of the null hypothesis of uncoupling estimated along the different causal directions. As depicted in Fig. 7(b), such a percentage is substantial for the coupled directions, while it is close to the expected type-I error probability for the uncoupled directions.

\section{E. Limits of applicability}

Since the proposed approach is directly based on the concept of Granger causality, its operational implementation is subject to restrictions and limitations of the causality definition, which were made explicit by Granger himself in his seminal papers $[4,28]$. One of these restrictions is related to the necessity of providing a causally complete description of the observed system, in order to avoid detection of spurious connections due to common sources or missing variables. Another axiom for Granger causality implies that any variable that is a perfect function of one or more other variables should be excluded from the observation set [28]. This case involves, e.g., fully synchronized systems, for which it is known that state space-based methods like transfer entropy [10] and predictability improvement $[17,18]$ cannot detect the presence of coupling because the driving and driven systems are indistinguishable to each other.

Like any other approach grounded on the notion of Granger causality, the proposed method fails to detect causality when the present state of the investigated process can be fully described from its own past states. In this case, the entropy of the driven process conditioned to its own past would be zero and thus could not be further reduced using data from the driving process, preventing the detection of the drive-response system coupling. To explore this situation in practical examples, we applied our approach to coupled deterministic and stochastic systems with different degrees of self-predictability of the observed dynamics. As deterministic and stochastic systems, we considered respectively two unidirectionally coupled Logistic maps [29],

$$
\begin{gathered}
x_{1, n}=\rho x_{1, n-1}\left(1-x_{1, n-1}\right), \\
x_{2, n}=C x_{1, n-1}+(1-C)\left[\rho x_{2, n-1}\left(1-x_{2, n-1}\right)\right],
\end{gathered}
$$

and two unidirectionally coupled autoregressive (AR) process fed with independent Gaussian white noises $w_{1}$ and $w_{2}$ [30]:

$$
\begin{gathered}
x_{1, n}=\sqrt{2} \rho x_{1, n-1}-\rho^{2} x_{1, n-2}+w_{1, n}, \\
x_{2, n}=\sqrt{2} \rho\left[C x_{1, n-1}+(1-C) x_{2, n-1}\right]-\rho^{2} x_{2, n-2}+w_{2, n} .
\end{gathered}
$$

In both simulations, $C$ represents the coupling strength from $x_{1}$ to $x_{2}$, while the parameter $\rho$ was varied to achieve different behaviors for the dynamical system. Increasing $\rho$ from 3.5 to 4 , the logistic maps in Eq. (11) move from a periodic and fully predictable regime to a chaotic, non-fully predictable regime. On the contrary, the AR processes in Eq. (12) become progressively more and more predictable when $\rho$ is increased from 0 to 0.98 . Results in Fig. 8 show, for values of $C$ set to get a detectable coupling from $x_{1}$ to $x_{2}$, the minimum corrected conditional entropy estimated for the driven series $x_{2}$ either excluding or including terms of the driving series $x_{1}$ in the set of initial candidate terms. Considering the deterministic system [Fig. 8(a)] we note that, for values of the control parameter preceding the onset of chaos $(\rho<3.57)$, the driven system is fully described from its own past states. In this condition, in which the logistic maps exhibit permanent oscillations of finite period [29], the minimum conditional entropy is zero both before and after consideration of the driving series, so that the causal coupling could not be determined. As soon as chaos sets in $(\rho>3.57)$, the driven system is no longer fully predictable using only its past terms; in this case, the minimum conditional entropy decreases when candidates from the driving system are considered, thus allowing the quantification of a positive causal coupling. If we consider the stochastic system [Fig. 8(b)], we see that the entropy of the driven series conditioned only to 

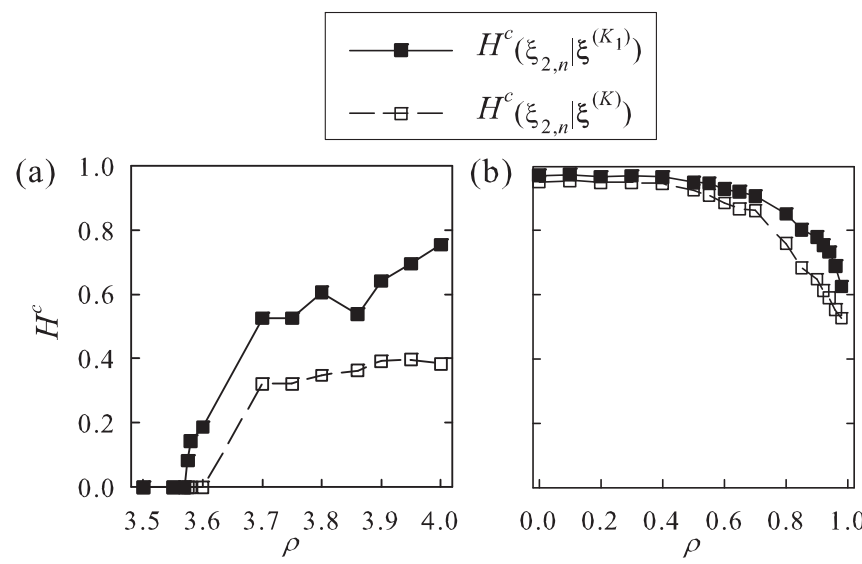

FIG. 8. Minimum corrected conditional entropy estimated, on the simulation of unidirectionally coupled Logistic maps (a) and AR processes (b), for the series $x_{2}$ when the set of initial candidate terms is $\Omega^{-2}$ (black squares) or $\Omega$ (white squares). All values in the plots are the average over 20 realizations of the simulation, and are expressed as a function of the parameter $\rho$ in Eqs. (11) and (12). Analysis parameters: $C=0.2$ for the Logistic system (a), $C=0.5$ for the AR system (b); $N=1000 ; Q=6 ; L=10$.

its own past never falls to zero, even for values of the control parameter approaching the condition of maximum regularity of the dynamics $(\rho=0.98)$. Correspondingly, the inclusion of terms from the driving series always leads to a reduction in the minimum conditional entropy, thus favoring the detection of the imposed causal coupling.

Another important aspect, which may affect the applicability of the proposed approach, is related to the dependence of the results on the parameters determining entropy estimation in practical time series of finite length. To assess such a dependence within the framework proposed in this study, we studied the effects of varying the number of quantization levels $Q$. Theoretically, increasing $Q$ would lead to finer partition of the state space, better estimates of conditional probabilities, and ultimately to more accurate coupling estimates. This observation holds for $N \rightarrow \infty$. In real applications the series length is finite and $Q$ should remain as low as $Q^{K} \approx N$ to guarantee a reliable approximation of probabilities with sample frequencies [19]. Figures 9 and 10 show the corrected conditional entropy and causal coupling estimated as a function of the coupling strength for different values of $Q$ and $N$ set for the simulations of Eqs. (11) and (12), respectively. As a general result, we see that the utilization of finer partitions yielded by increasing $Q$ brings about an increase of corrected conditional entropy estimates, as well as a decrease in the gap between entropies estimated, for the driven series, excluding and including the driving series in the analysis (Figs. 9 and 10 , left columns). This second result leads to estimates of the causal coupling that decrease progressively at increasing the number of quantization levels (Figs. 9 and 10, right columns). As expected, these effects are dependent on the time series length, in such a way that if $N$ is higher the causal coupling may be detected for higher values of $Q$. This result is related to the fact that probabilities in higher dimensional state spaces are estimated with progressively increasing accuracy for longer time series.

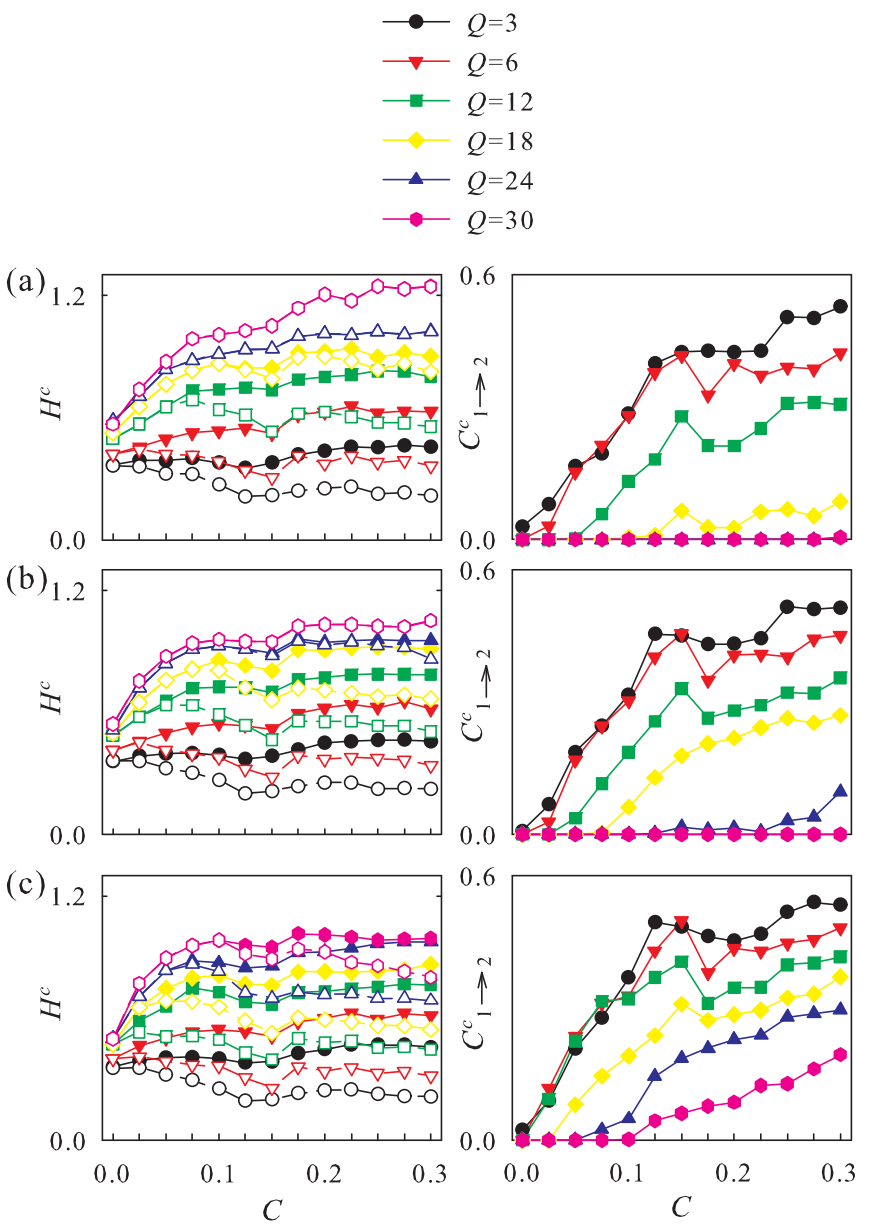

FIG. 9. (Color online) Dependence on the number of quantization levels $Q$ used to estimate conditional entropy for the simulation of unidirectionally coupled Logistic maps with $\rho=3.8$. Plots depict the minimum corrected conditional entropy for the series $x_{2}$ when the set of initial candidate terms is $\Omega^{-2}$ (filled symbols) or $\Omega$ (empty symbols) (left panels), together with the corresponding causal coupling (right panels), averaged over 20 simulation runs and expressed as a function of the coupling parameter $C$ for time series length $N=300$ (a), $N=500$ (b), and $N=1000$ (c).

\section{EVALUATION ON PHYSIOLOGICAL SYSTEMS}

This section describes the evaluation of the proposed approach for quantifying nonlinear Granger causality in physiological systems where commonly only short time series (few hundred points) are available due to stationarity constraints. The considered applications are the study of short-term interactions among cardiovascular and cardiorespiratory variability series, and the study of propagation of the electrocortical activity of the brain measured from multichannel electroencephalographic (EEG) recordings. We apply the nonuniform embedding procedure quantizing the physiological time series with $Q=6$ quantization levels and using $L=10$ as maximum lag for the candidate terms.

\section{A. Cardiovascular and cardiorespiratory interactions}

As a first practical application, we study nonlinear causality in short-term cardiovascular and cardiorespiratory 

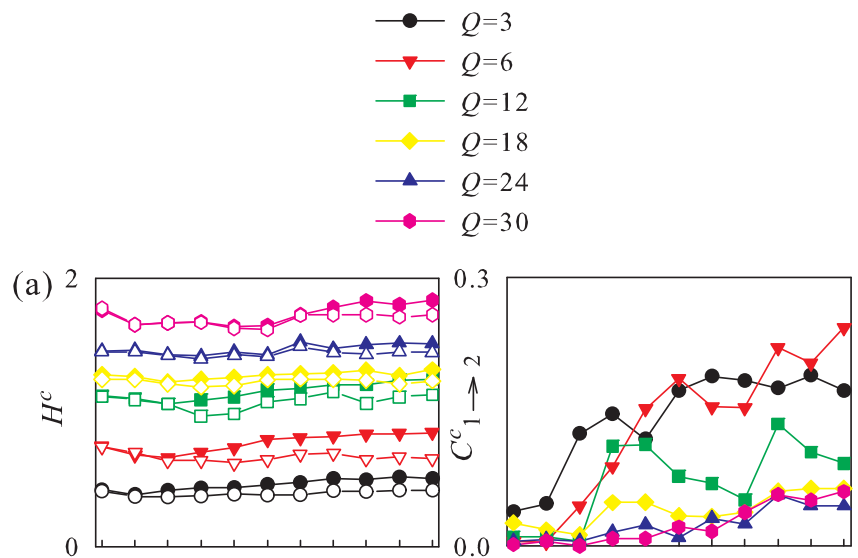

(b)
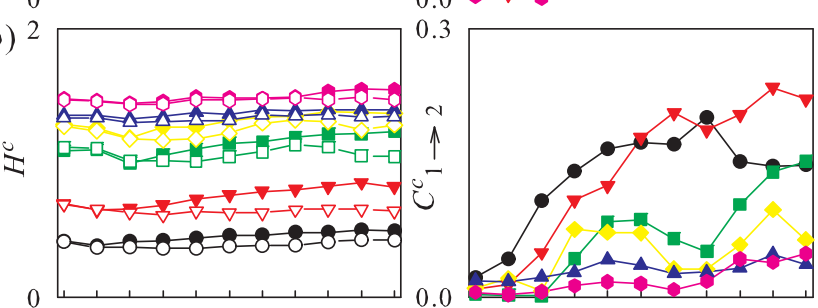

(c)
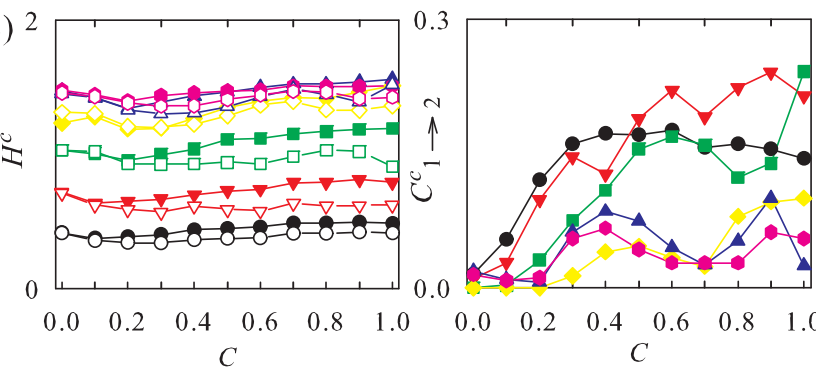

FIG. 10. (Color online) Dependence on the number of quantization levels $Q$ used to estimate conditional entropy for the simulation of unidirectionally coupled AR processes with $\rho=0.94$. Plots and symbols are as in Fig. 9.

interactions. The considered dynamical systems are the respiratory system, the vascular system, and the cardiac system, which we denote as $X_{1}, X_{2}$, and $X_{3}$, respectively. The acquired signals are noninvasive recordings of electrocardiogram (ECG, lead II), finger photopletismographic arterial pressure (Finapres device) and respiratory nasal flow (by differential pressure transducer) obtained in a young healthy subject (20 years old) in sinus rhythm and breathing spontaneously [31]. From these signals, the beat-to-beat time series of the heart period $\left(x_{3, n}\right)$, systolic arterial pressure $\left(x_{2, n}\right)$, and respiratory flow $\left(x_{1, n}\right)$ are offline measured, respectively, as the sequences of the temporal distances between consecutive $R$ waves of the ECG, the local maxima of the arterial pressure signal inside each detected heart period, and the values of the respiratory signal taken at the onset of each heart period. Two stationary, artifact-free windows of $N=300$ samples, measured synchronously for the $M=3$ time series, are considered for the analysis, the first in the resting supine position and the second in the upright position assumed by the subject after passive head-up tilting. As the adopted measurement convention allows instantaneous (i.e., not delayed) effects from respiration to systolic pressure and to heart period, as well as from systolic pressure to heart period, we include the zero-lag term in the set of initial candidate components when appropriate; for instance, the set $\Omega=$ $\left\{x_{1, n}, x_{1, n-1}, \ldots, x_{1, n-10}, x_{2, n}, x_{2, n-1}, \ldots, x_{2, n-10}, x_{3, n-1}, \ldots\right.$, $\left.x_{3, n-10}\right\}$, including the zero-lag terms $x_{1, n}$ and $x_{2, n}$ in addition to the lagged terms, is considered for the analysis of causality from $X_{1}$ to $X_{3}$.

The results of the analysis are reported in Fig. 11. In each plot, results of the description of the target series $x_{i}$ are shown for the procedure applied either excluding or including in the initial set of candidate components the terms of the input series $x_{j}$, yielding, respectively, the black and red (gray triangles) curves. A difference between the two curves is observed only if some terms from the input system are selected for the embedding of the target system, leading to a decrease in the corrected conditional entropy and thus to a positive value of the causal coupling. In the supine position [Fig. 11(a)], this situation occurs from $X_{1}$ to $X_{2}$ and to $X_{3}$, and from $X_{3}$ to $X_{2}$, with causal coupling values detected as significant in accordance with the test based on surrogate data ( $S=40$ surrogates, significance $\alpha=0.0163$ ). The opposite situation, with terms from the input system not selected even when available in the set of candidates, leads to unaltered conditional entropy estimates. This occurs from $X_{2}$ to $X_{1}$, from $X_{3}$ to $X_{1}$, and from $X_{2}$ to $X_{3}$, with coupling equal to zero (and nonsignificant). The overall picture is in agreement with behaviors that are well explainable in terms of the known cardiovascular physiology: The unidirectional interactions from $X_{1}$ to $X_{2}$ and from $X_{1}$ to $X_{3}$ document expected physiological mechanisms whereby respiration affects both the arterial pressure, through mechanical effects, and the heart rate, according to the so-called respiratory sinus arrhythmia phenomenon [32]; moreover, the significance of the coupling from $X_{3}$ to $X_{2}$ and the simultaneous absence of coupling in the opposite direction from $X_{2}$ to $X_{3}$ are in agreement with the notion that mechanisms operating in the direction from heart period to arterial pressure may prevail, in the nonsolicited resting condition of the subject, over the baroreflex mechanism describing driving effects from systolic pressure to heart period [33,34]. Results obtained in the upright position after head-up tilt evidence an alteration of the Granger causal relationships among the observed systems [Fig. 11(b)]. While the mechanical effects of respiration on systolic pressure are still described (the coupling from $X_{1}$ to $X_{2}$ is significant, $\alpha=0.0405$ ), the respiratory sinus arrhythmia mechanism from respiration to heart period is dampened (the coupling from $X_{1}$ to $X_{3}$ is null) as a consequence of the shift of the cardiovascular sympathovagal balance toward sympathetic activation and vagal deactivation provoked by tilt [35]. In addition, the emergence of a strong coupling from $X_{2}$ to $X_{3}$ (significance $\alpha=0.0163$ ), together with the corresponding decrease of coupling from $X_{3}$ to $X_{2}$ (significance $\alpha=0.0647$ ), document an enhanced regulation over the baroreflex pathway consequent to the continuous solicitation of the sympathovagal balance resulting from the assumption of the upright position [34-36].

\section{B. EEG interactions}

As a second practical example, the method is applied on EEG recordings measured from different cortical locations in 

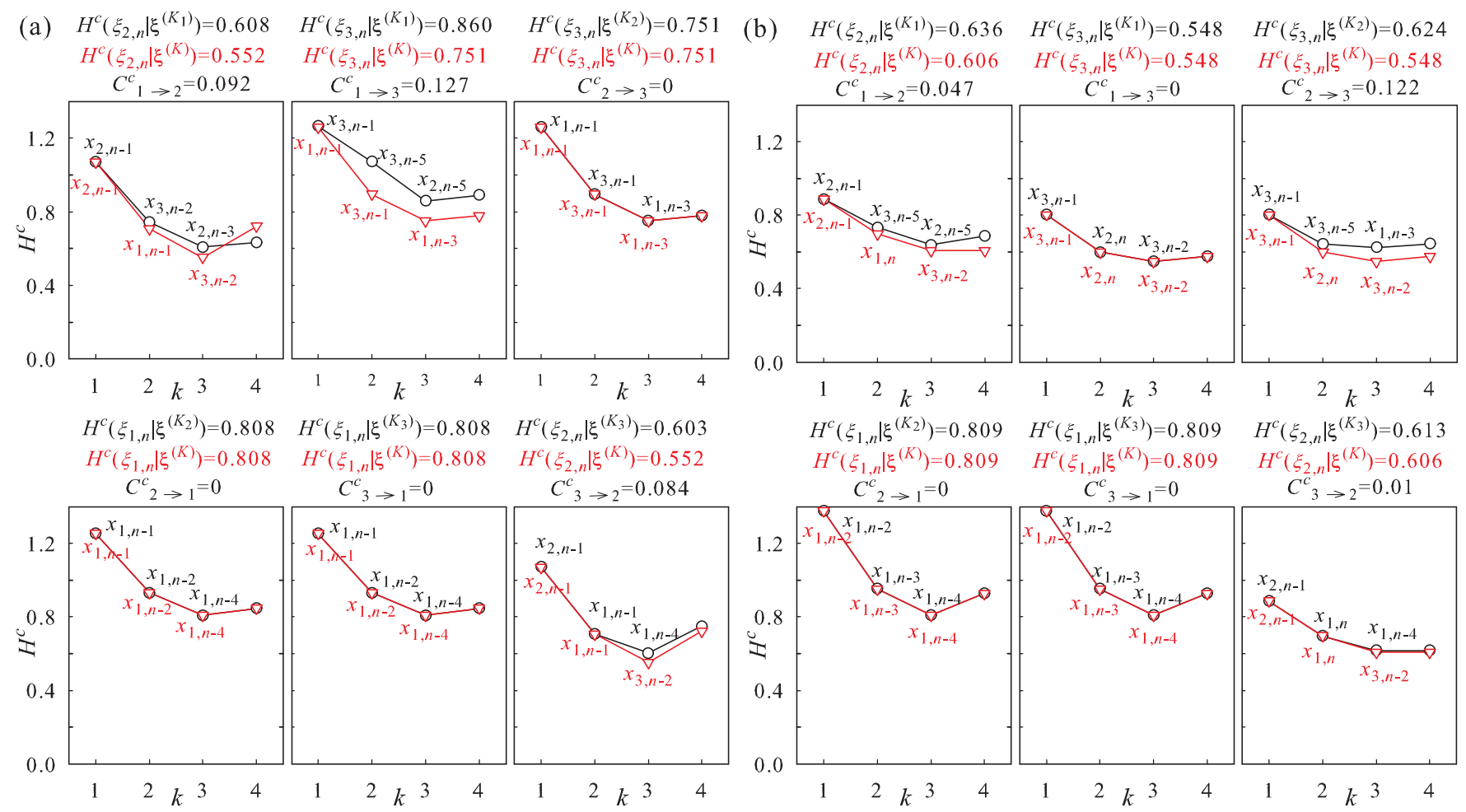

FIG. 11. (Color online) Application of the procedure for nonuniform multivariate embedding to respiratory flow $\left(x_{1}\right)$, systolic arterial pressure $\left(x_{2}\right)$, and heart period $\left(x_{3}\right)$ time series measured in the supine position (a) and in the upright position (b). Each plot depicts the corrected conditional entropy of $X_{i}$ when the set of initial candidate terms is $\Omega^{-j}$ (black circles) or $\Omega$ [red (gray) triangles], estimated as a function of the dimension $k$ of the embedding vector. The candidate term selected at each step of the procedure in indicated in the plot.

a young healthy subject (27 years old) resting in the relaxed awake state, both during eyes closed and eyes open conditions [37]. We consider multichannel EEG recorded according to the international 10-20 system (Fpz common reference), with $256-\mathrm{Hz}$ sampling rate. As preprocessing steps, signals are band-pass filtered (Fast Fourier Transform filter, 0-40 Hz) and downsampled to $64 \mathrm{~Hz}$. Different cortical areas are considered selecting appropriate electrode locations $(M=$ 4): $X_{1}$, posterior area (electrode $\mathrm{Pz}$ ); $X_{2}$, left central area (electrode C3); $X_{3}$, right central area (electrode C4); $X_{4}$, frontal area (electrode Fz). In order to reduce the effects of the reference electrode location, the signal to be analyzed for each area is obtained subtracting from the signal measured at the considered electrode the average signal of its four nearest electrodes, according to the Hjorth surface Laplacian technique [38]. Two artifact-free windows of $8 \mathrm{~s}$ duration ( $N=512$ samples), in the eyes closed and eyes open conditions, are then selected for the analysis.

Figure 12 reports the causal coupling estimated between each pair of time series in the two conditions. During eyes closed, nonzero values of causal coupling are observed from the posterior area towards all other cortical areas, and from the left central to the right central areas [Fig. 12(a)]. This pattern of $C_{i \rightarrow j}^{c}$ values suggests that the EEG activity in this condition propagates mainly along a back-to-front direction, while front-to-back propagation is absent. The result is strengthened by the statistical analysis, showing that the link is significant over all directions for which the coupling value is nonzero. This behavior is likely related to the presence of a dominant $\alpha$ activity in subjects with eyes closed [39]. This activity is supposed to originate in the occipital visual cortex, located in proximity of the posterior brain areas, and then to spread toward the central and anterior brain areas [40]. Another interesting result is that the stronger coupling is that from the posterior to the frontal areas, both in absolute value $\left(C_{1 \rightarrow 4}^{c}=\right.$ 0.088 ) and in statistical significance (the original coupling value is larger than any surrogate value). The observation that $\alpha$ activity is more coupled between posterior and anterior cortical regions than between central and other regions was reported in early studies [41]. Besides the back-to-front EEG propagation, a significant interaction from $X_{2}$ to $X_{3}$ is also observed. This observation is in agreement with previous results $[37,42]$ showing that in the eyes closed condition the sources of EEG activity are mainly located in the left occipital areas, and propagate in the forward direction but also toward the right hemisphere. The results obtained with eyes open, reported in Fig. 12(b), are less easy to interpret because the literature is mostly focused on studies of traveling waves of the $\alpha$ activity, while the $\alpha$ rhythm is known to weaken with the opening of the eyes. Multivariate embedding procedures like ours provide a nonlinear measure of Granger causality which is not restricted to specific frequency bands, but rather reflects the interaction between the overall dynamics of the two considered subsystems. Hence the generally low values observed for causal coupling, with statistical significance reached in only one causal direction, are likely to indicate the presence of a pattern of EEG signals in which multiple and/or irregular rhythms do not exhibit a consistent direction of propagation. 

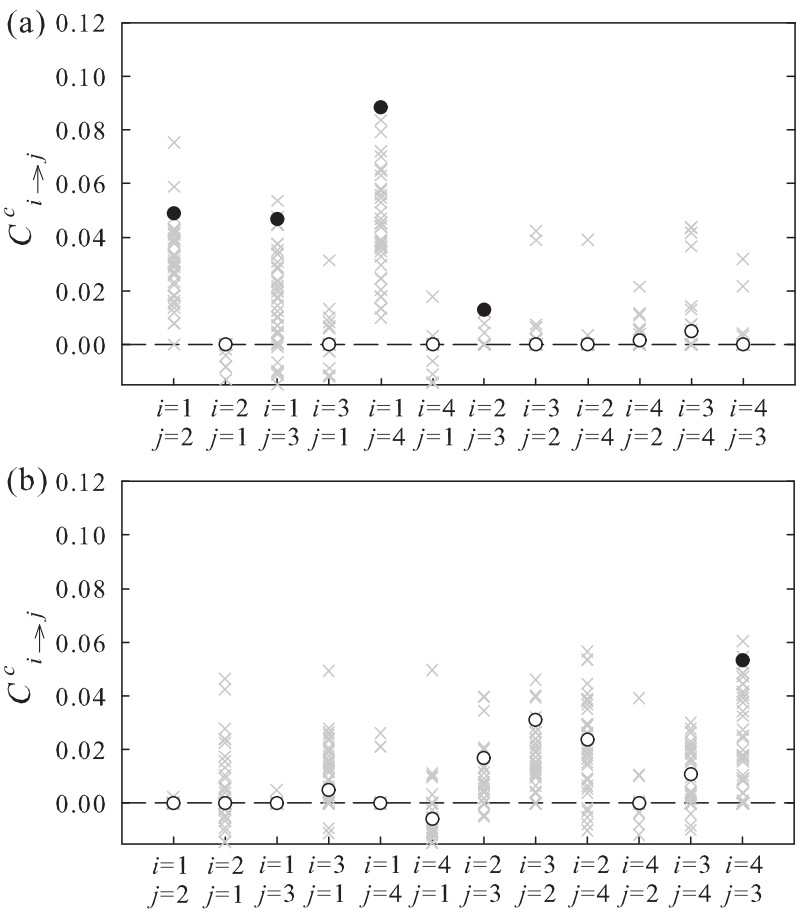

FIG. 12. Application of the procedure for nonuniform multivariate embedding to EEG signals measured with eyes closed (a) and with eyes open (b). Each plot depicts the estimated causal coupling from $X_{i}$ to $X_{j}$ evaluated for the original time series (circles) and for the set of $S=40$ surrogate time series (gray crosses). Filled circles represent causal coupling values detected as statistically significant (significance: $\alpha=0.0647$ ). $X_{1}=$ posterior; $X_{2}=$ left central; $X_{3}=$ right central; $X_{3}=$ frontal.

\section{DISCUSSION}

We have presented a model-free, information theoretic tool for the assessment of nonlinear Granger causality from multiple interacting dynamical systems. Being grounded on information theory, the method does not make strong assumptions about the nature of the investigated dynamics, and thus works both for deterministic and stochastic systems. This property favors utilization on physiological time series in which the type of the dynamics cannot be assumed a priori. The approach was devised to cope with problems typical of application of information-theoretic tools on multiple experimental time series, such as the bias affecting estimation of the conditional entropy at increasing the dimensionality of the embedding and/or decreasing the length of the available data.

The main peculiarity of the approach is the procedure devised for nonuniform embedding of multiple time series. Such a procedure allows an intuitive selection of the terms to be included in multivariate embedding, based on the fact that only the candidate components which contribute most to the prediction of the target series are allowed to enter-in a progressive fashion - the embedding vector. Unlike traditional uniform embedding schemes where components from all series are included in multivariate embedding vectors, in our nonuniform embedding scheme the components are selected only if they are useful for prediction. We have demonstrated the superiority of nonuniform embedding in the detection of Granger causality, showing that the arbitrariness inherent to uniform embedding may mask the detection of weak coupling conditions. Moreover, this feature allows us to control overfitting and, ultimately, to limit the rate of false causality detection. In fact we found that, considering the situations in which absence of coupling was imposed from one system to another in our simulation examples, in the large majority of cases no one component of the input system was included in the embedding vector, so that the procedure returned causal coupling equal to zero along the uncoupled directions. Few exceptions of input components entering the embedding vector in uncoupled directions were observed for very short or noisy time series. These situations led to negative or slightly positive values of the causal coupling that resulted in being undistinguishable from those obtained for input surrogate time series where components enter the embedding vector only by chance.

The second important aspect is the utilization of the corrected conditional entropy estimator. By compensating the bias that affects the conditional entropy estimates at increasing the embedding dimension, the corrected estimator serves to provide an objective criterion, i.e., the corrected conditional entropy minimum, for the termination of the embedding procedure. As also seen in the investigated simulated systems, the empirical correction proposed here does not guarantee to retrieve the correct embedding dimension; this appears too demanding a task to be achieved from short and noisy data realizations. However, we found that this fact does not affect remarkably the detection of Granger causality and the quantification of the coupling strength. In the absence of coupling, the estimated embedding dimension was small enough to avoid the inclusion of unwanted input components which could give rise to spurious causality; in the presence of coupling, it was large enough to allow inclusion of relevant input components, which made the causal coupling differ significantly from zero. As expected, both specificity and sensitivity degraded with shortening of the available data sequences and with noise contamination. Nevertheless, the approach allowed reliable rejection and detection of causality in the conditions typical of experimental time series (few hundred points available and limited noise corruption). In these conditions, both the causal coupling values and the rate of causality detection were found to increase with the coupling parameter set in the simulations.

The limits of applicability of the method were investigated evaluating its ability to detect coupled states either for different types of dynamical systems, or for different values of the analysis parameters. In general terms, the approach does not work whenever the driven process is fully predictable without any need of using samples from the driving signal. We found that this is the case for deterministic nonchaotic systems in which the present state of the driven process is functionally related to its past states and/or to the past states of processes other than the driving one. In such a case, no causal interaction could be detected by any method based on Granger causality because the full description of the driven system is obtained already before incorporating information from the driving system. On the contrary, we showed applicability of the method for stochastic systems, where the intrinsic nature of the observed dynamics does not allow the corrected conditional entropy to 
decay to zero, as well as for deterministic chaotic systems, where the evolution from similar states cannot be fully predicted. Applicability was demonstrated for a broad range of simulated deterministic chaotic systems or stochastic systems, ranging from continuous to discrete systems, from bivariate to multivariate and spatially extended processes, over realizations of different length and using different values for the analysis parameters. We ascribe the ability of the approach to detect the information transfer in such a broad range of situations to the progressive nature of the proposed embedding scheme and to the strict threshold implicitly set by the conditional entropy estimator, which limits the number of components selected by the procedure to those effectively important for the description of the observed dynamics. For instance, in deterministic systems an embedding scheme working on the driven variable only would be theoretically as good as a scheme involving both driven and driving variables, provided that the embedding dimension is sufficiently high. Since the proposed procedure prevents the inclusion of redundant components into the embedding vector, it realizes a parsimonious approach to the reconstruction of the state space, seeking the lowest dimension of the reconstructed space that allows the best predictability of the driven dynamics. This feature is helpful to improve causality estimation and definitely contributes to the efficacy of the proposed method. This way to proceed shares some similarities with a very recently proposed method using nonuniform embedding and an arbitrary threshold selection on conditional mutual information for estimating directional coupling in bivariate deterministic systems [16].

The approach has been shown to be useful in the description of physiological systems composed of multiple interacting subsystems, such as the cardiovascular and cardiorespiratory ones, and of spatially extended physiological systems, such as the cortical system where EEG activity is supposed to propagate among different scalp locations. We emphasize that here a preliminary analysis was performed to verify the feasibility of the approach in different fields of application, and that systematic tests performed on extensive databases should be carried out to corroborate the validity of the results. Nevertheless, we observed patterns of Granger causality which agree with known mechanisms of cardiovascular physiology and neural physiology; significant examples are the emergence of causality from arterial pressure to heart period variability with the assumption of the upright position, documenting an increased activity of the baroreflex regulation of heart rate, and the presence of causality from the posterior towards the central and anterior EEG recorded during eyes closed wakefulness, suggesting a back-to-front propagation of the brain $\alpha$ waves.
[1] L. Faes and G. Nollo, Med. Biol. Eng. Comput. 44, 383 (2006); G. Nollo et al., Am. J. Physiol. 283, H1200-H1207 (2002); A. Porta, F. Aletti, F. Vallais, and G. Baselli, Philos. Trans. R. Soc., A 367, 391 (2009); M. G. Rosenblum, L. Cimponeriu, A. Bezerianos, A. Patzak, and R. Mrowka, Phys. Rev. E 65, 041909 (2002); C. Schafer, M. G. Rosenblum, J. Kurths, and H. H. Abel, Nature (London) 392, 239 (1998); A. Stefanovska, H. Haken, P. V. E. McClintock, M. Hozic, F. Bajrovic, and S. Ribaric, Phys. Rev. Lett. 85, 4831 (2000).

[2] E. Pereda, R. Quian Quiroga, and J. Bhattacharya, Prog. Neurobiol. 77, 1 (2005).

[3] N. Wiener, The Theory of Prediction (McGraw-Hill, New York, 1956), Chap. 8.

[4] C. W. J. Granger, Econometrica 37, 424 (1969).

[5] L. A. Baccala and K. Sameshima, Biol. Cybern. 84, 463 (2001); M. Kaminski, M. Ding, W. A. Truccolo, and S. L. Bressler, ibid. 85, 145 (2001).

[6] N. Ancona, D. Marinazzo, and S. Stramaglia, Phys. Rev. E 70, 056221 (2004); D. Marinazzo, M. Pellicoro, and S. Stramaglia, ibid. 73, 066216 (2006); M. Riedl et al., Philos. Trans. R. Soc., A 367, 1407 (2009).

[7] K. Hlavackova-Schindler, M. Palus, M. Vejmelka, and J. Bhattacharya, Phys. Rep. 441, 1 (2007).

[8] A. Porta et al., Biol. Cybern. 81, 119 (1999).

[9] A. Papoulis, Probability, Random Variables and Stochastic Processes (McGraw-Hill, New York, 1984).

[10] T. Schreiber, Phys. Rev. Lett. 85, 461 (2000).

[11] M. Palus, V. Komarek, Z. Hrncir, and K. Sterbova, Phys. Rev. E 63, 046211 (2001).

[12] M. Palus and M. Vejmelka, Phys. Rev. E 75, 056211 (2007).

[13] D. Marinazzo, M. Pellicoro, and S. Stramaglia, Phys. Rev. Lett. 100, 144103 (2008); Phys. Rev. E 77, 056215 (2008).
[14] L. Barnett, A. B. Barrett, and A. K. Seth, Phys. Rev. Lett. 103, 238701 (2009).

[15] M. Chavez, J. Martinerie, and M. Le Van Quyen, J. Neurosci. Methods 124, 113 (2003); V. A. Vakorin, O. A. Krakovska, and A. R. McIntosh, ibid. 184, 152 (2009); P. F. Verdes, Phys. Rev. E 72, 026222 (2005).

[16] I. Vlachos and D. Kugiumtzis, Phys. Rev. E 82, 016207 (2010).

[17] U. Feldmann and J. Bhattacharya, Int. J. Bifurcation Chaos 14, 505 (2004); M. C. Romano, M. Thiel, J. Kurths, and C. Grebogi, Phys. Rev. E 76, 036211 (2007).

[18] L. Faes, A. Porta, and G. Nollo, Phys. Rev. E 78, 026201 (2008).

[19] A. Porta et al., Biol. Cybern. 78, 71 (1998).

[20] H. Akaike, IEEE Trans. Autom. Control 19, 716 (1974); J. Rissanen, Ann. Stat. 11, 417 (1983).

[21] F. Takens, Lect. Notes Math. 898, 366 (1981)

[22] R. Q. Quiroga, A. Kraskov, T. Kreuz, and P. Grassberger, Phys. Rev. E 65, 041903 (2002).

[23] G. H. Yu and C. C. Huang, Stoch. Env. Res. Risk Ass. 15, 462 (2001).

[24] M. Le Van Quyen, J. Martinerie, C. Adam, and F. J. Varela, Physica D 127, 250 (1999).

[25] R. Quian Quiroga, J. Arnhold, and P. Grassberger, Phys. Rev. E 61, 5142 (2000).

[26] G. V. Osipov, B. Hu, C. Zhou, M. V. Ivanchenko, and J. Kurths, Phys. Rev. Lett. 91, 024101 (2003).

[27] M. Wiesenfeldt, U. Parlitz, and W. Lauterborn, Int. J. Bifurcation Chaos 11, 2217 (2001).

[28] C. W. J. Granger, J. Econ. Dynam. Control 2, 329 (1980).

[29] R. M. May, Nature (London) 261, 459 (1976).

[30] L. Faes, G. Nollo, and K. H. Chon, Ann. Biomed. Eng. 36, 381 (2008).

[31] L. Faes et al., Biol. Cybern. 90, 390 (2004). 
[32] R. W. deBoer, J. M. Karemaker, and J. Strackee, Am. J. Physiol. 253, H680 (1987); J. P. Saul, R. D. Berger, M. H. Chen, and R. J. Cohen, ibid. 256, H153 (1989).

[33] T. J. Mullen et al., Am. J. Physiol. 272, H448 (1997).

[34] G. Nollo et al., Am. J. Physiol. 288, H1777 (2005).

[35] A. Malliani, News Physiol. Sci. 14, 111 (1999).

[36] N. Montano et al., Circulation 90, 1826 (1994).

[37] L. Faes, A. Porta, and G. Nollo, IEEE Trans. Biomed. Eng. 57, 1897 (2010).
[38] B. Hjorth, Electroencephalogr. Clin. Neurophysiol. 39, 526 (1975).

[39] C. Babiloni et al., Hum. Brain Mapp. 27, 162 (2006).

[40] P. L. Nunez, IEEE Trans. Biomed. Eng. 21, 473 (1974).

[41] J. Ito, A. R. Nikolaev, and C. van Leeuwen, Biol. Cybern. 92, 54 (2005); H. Ozaki and H. Suzuki, Electroencephalogr. Clin. Neurophysiol. 66, 191 (1987).

[42] M. Kaminski, K. Blinowska, and W. Szclenberger, Electroencephalogr. Clin. Neurophysiol. 102, 216 (1997). 